\title{
Synthesis of amino-methylene-gem-bisphosphonates containing an aziridine motif: Studies of the reaction scope and insight into the mechanism
}

\author{
Thomas Cheviet, ${ }^{[\mathrm{a}]}$ and Suzanne Peyrottes*[a]
}

Team Nucleosides \& Phosphorylated Effectors, Institute for Biomolecules Max Mousseron (IBMM), UMR 5247 CNRS, ENSCM, Univ. Montpellier, Campus Triolet, cc1705, Place Eugène Bataillon, 34095 Montpellier, France

Corresponding author's e-mail: suzanne.peyrottes@ umontpellier.fr

\section{Table of Contents/Abstract Graphic}

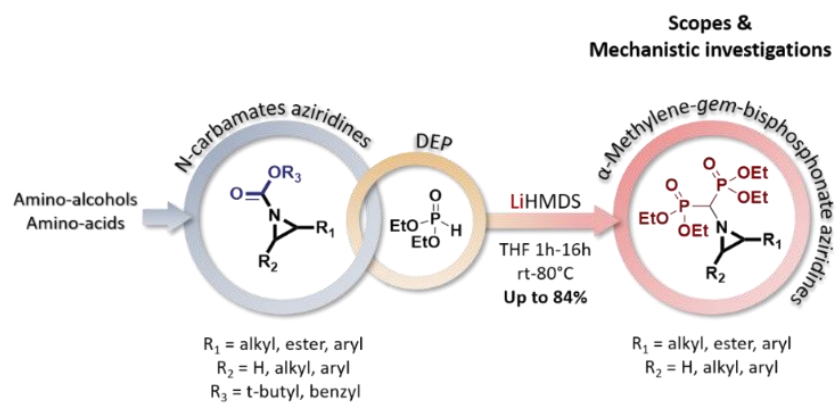

\begin{abstract}
A broad range of $\mathrm{N}$-carbamoyl aziridines were obtained and then treated by the diethyl phosphonate anion to afford $\alpha$-methylene-gem-bisphosphonate aziridines. Study of the reaction's scope and additional experiments indicate that the transformation proceed via a new mechanism involving the chelation of lithium ion. This last is crucial for the reaction to occur and disfavors the aziridine ringopening. A phosphonate-phosphate rearrangement from a $\alpha$-hydroxybisphosphonate aziridine intermediate is also proposed for the first time. This reaction provides a simple and convenient method for the synthesis of highly functionalized phosphonylated aziridine motif.
\end{abstract}




\section{INTRODUCTION}

Aziridines are widely studied as key intermediates in organic syntheses and represent a valuable source of chiral centers because of their regio- and stereo-selectivity in ring-opening reactions. Their reactivity has recently been reviewed. ${ }^{1}$ When combined with a phosphonate moiety (widely used in medicinal chemistry as bio-isoster of organic phosphate and insensitive to enzymatic cleavage), they are useful building blocks for the synthesis of amino-phosphonates that can be considered as analogues of $\alpha$-aminoacids. As example, the synthesis of aziridine-2-phosphonates and aziridine-1-ylmethylphosphonates have been well described in the literature. ${ }^{2-5}$ However, the association of a chiral aziridine with several phosphonate groups is rather limited and may lead to promising scaffolds for obtaining biomolecules of interest. Indeed, bisphosphonates (or BPs, Figure 1) have been developed for their coordination abilities and their structural analogy with inorganic pyrophosphate (the P-C-P bridge replacing the $\mathrm{P}-\mathrm{O}-\mathrm{P}$ bonds is not sensitive to enzymatic cleavage), making them suitable for the treatment of bone diseases or drug delivery. ${ }^{6-11} \mathrm{~N}$-BPs are nitrogen-containing bisphosphonates, targeting enzymes of the mevalonate pathway and exhibiting antiosteoporosis and antiproliferative activities. $^{12,13}$ For example, the incadronate (Figure 1) is an anti-osteoporosis drug with a 1aminobisphosphonate motif.

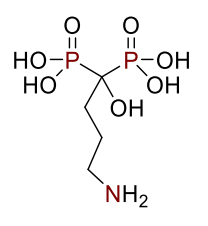

Alendronate

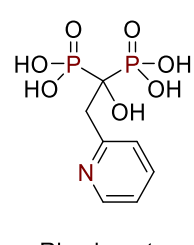

Risedronate

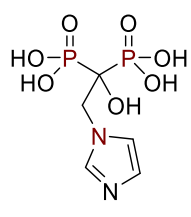

Zoledronate

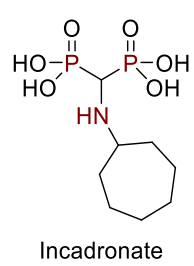

Incadronate

Figure 1. Structures of BPs and N-BPs clinically used or of biological interest.

Syntheses of 1-aminobisphosphonates (or aminomethylene-gem-bisphosphonates) are described through numerous methods, ${ }^{14}$, namely the condensation of ethyl orthoformates and phosphites, ${ }^{15,16}$ the Beckmann rearrangement of oximes, ${ }^{17,18}$ or the bisphosphorylation of amides, ${ }^{19,20}$ imines, ${ }^{21}$ isonitriles, ${ }^{22-24}$ or nitriles. ${ }^{25}$ To our knowledge, such derivatives when containing an aziridine motif are not reported in the literature. Thus, we propose an original synthetic route involving lithiated diethylphosphite for the valorization of these three-membered rings in phosphorus chemistry (Scheme 1). We use N-carbamoyl aziridines as starting material for the preparation of brand new phosphonylaziridine compounds, such as aziridine $\alpha$-methylene-gem-bisphosphonates (abbreviated AzbisPs). To investigate the scope and limitations of the method, a library of $\mathrm{N}$-carbamoyl aziridines is obtained beforehand. In addition, complementary experiments are performed to propose a mechanism for the formation of such derivatives. 


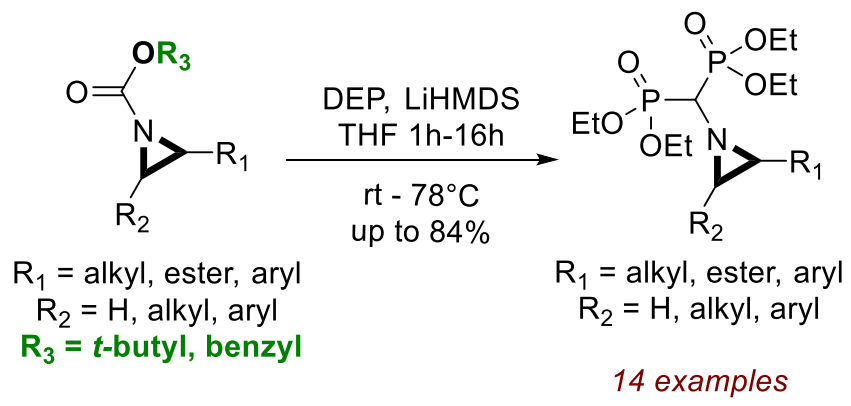

Scheme 1. Synthesis of aziridine $\alpha$-methylene-gem-bisphosphonates (AzbisPs) from Ncarbamoyl aziridines.

\section{RESULTS AND DISCUSSION}

In the course of a medicinal project, we envisaged the preparation of $\beta$-aminophosphonates through the ring-opening of aziridines with phosphites, ${ }^{26}$ a methodology poorly reported in the literature. ${ }^{27,28}$ When such conditions (DEP/LiHMDS in THF) are applied to a $\mathrm{N}-\mathrm{Cbz}$ aziridine as substrate, we observed the formation of an aziridine $\alpha$-methylene-gem-bisphosphonate (AzbisP), instead of the expected ring-opened product. To study the scope of this reaction, we examined the behavior of several $\mathrm{N}$-Boc or N-Cbz-protected aziridines.

With this aim, 14 substrates (Table 1, compounds 2a-n) were synthesized starting from commercially available amino-acids and amino-alcohols, through protection/reduction steps and then cyclization. Among them, nine N-carbamoyl aziridines $(\mathbf{2 a}, \mathbf{2 d}, \mathbf{2 e}, \mathbf{2 f}, \mathbf{2 g}, \mathbf{2 i}, \mathbf{2 j}, \mathbf{2 l}$ and $2 \mathbf{n})$ were hitherto unknow compounds, that may be viewed as valuable building blocks. According to the nature of the substituents, either Mitsunobu conditions (route A) or intramolecular substitution (route B) is used. Likely due to interactions/steric hindrance between the tosylate group and the phenyl moiety of the $\mathrm{Cbz}$ protecting group, the one-pot cyclisation of $\mathrm{N}-\mathrm{Cbz}$ aziridines usually led to lower yields than under Mitsunobu conditions. Except in the case of the cyclohexyl aziridine 2k (Table 1, entry 11), where the 3D conformation of the carbocycle is supposed to reduce the possible interactions between the two groups. We observed lower yields for compounds $\mathbf{2} \mathbf{I}$ and $\mathbf{2 m}$ (obtained as invertomers because of the high-energy barrier of the nitrogen inversion) and this may be associated with the formation of a highly constrained aziridine ring. In the Mitsunobu conditions, no reactivity is observed. 
Table 1. Preparation of $N$-carbamoyl aziridines (as substrates) from commercially available amino acids and amino-alcohols.

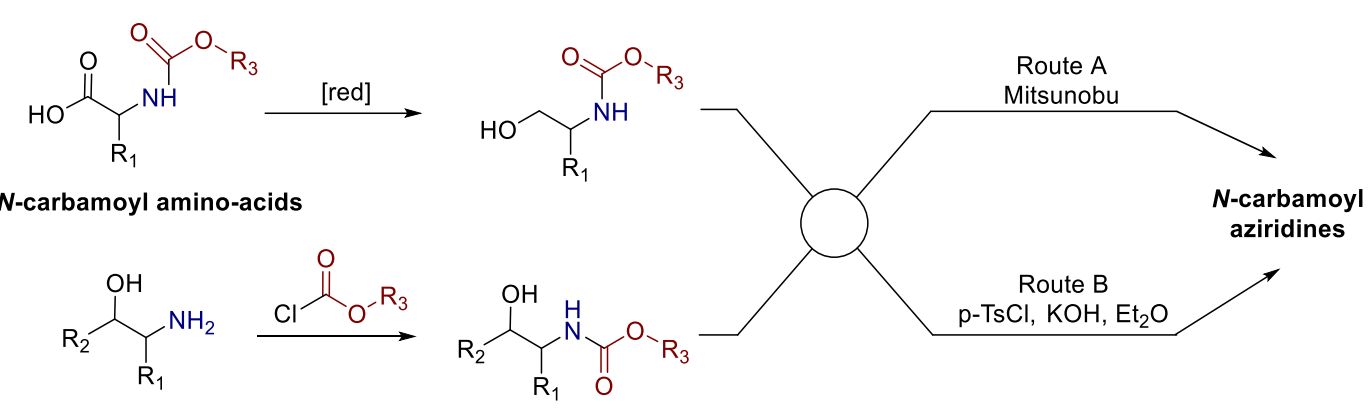

Amino-alcohols

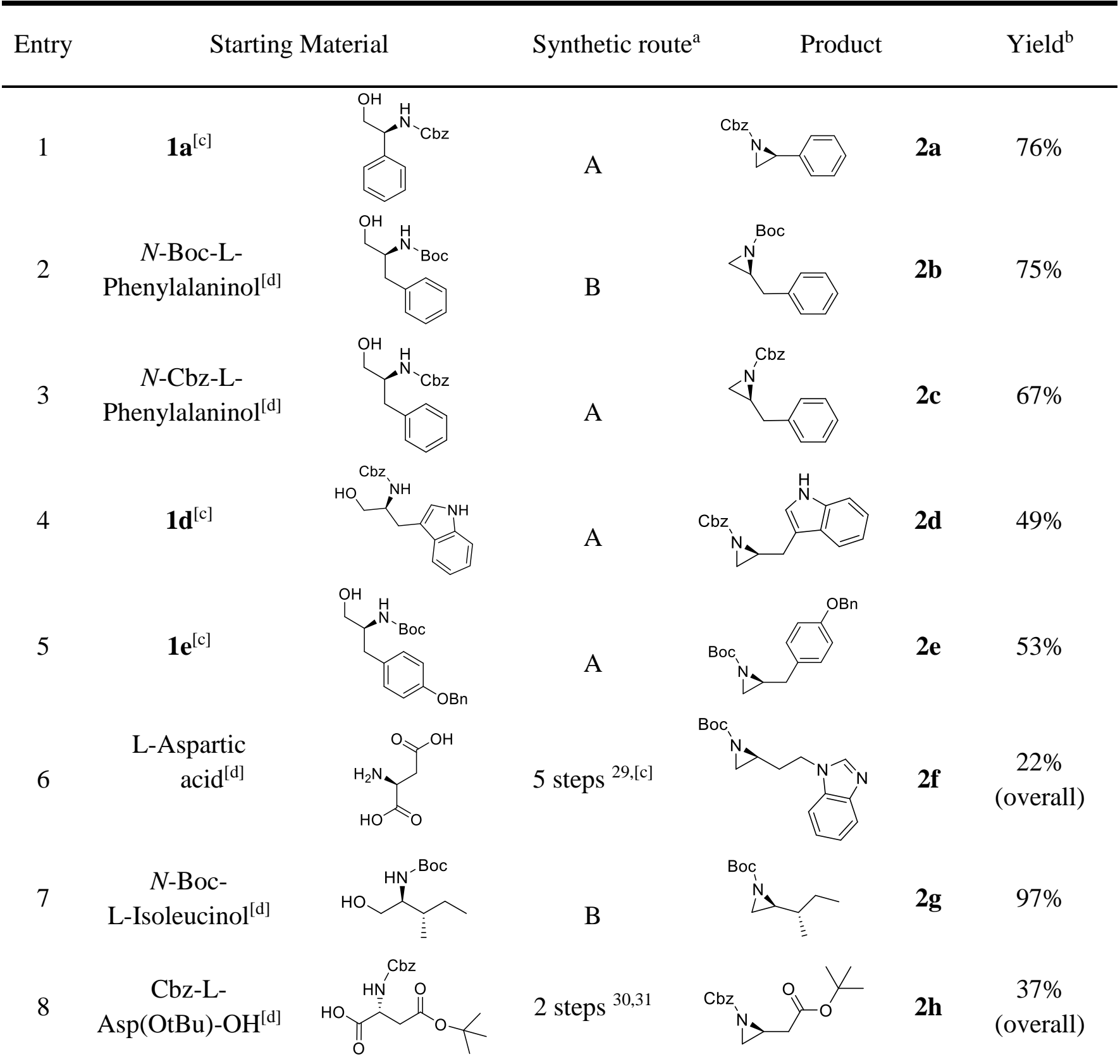




12

[a] Reaction conditions: Mitsunobu (route A) $\mathrm{PPh}_{3}, \mathrm{DEAD}, \mathrm{THF}, 0^{\circ} \mathrm{C}$ to rt, overnight; One-pot cyclisation (route $\mathrm{B}$ ) $p$ - $\mathrm{TsCl}, \mathrm{KOH}, \mathrm{Et}_{2} \mathrm{O}$, reflux, overnight. [b] Isolated yield after purification by column chromatography. [c] Preparation of starting material 1a, 1d, $\mathbf{1 e}$ and 1i-n is available in the experimental section. [d] Commercially available.

Having established the suitable reaction conditions (data not shown), aziridines 2a-n were treated with 6.1 equivalents of diethylphosphite and 6 equivalents of LiHMDS (1M in THF), in anhydrous $\mathrm{THF}$, at $-78^{\circ} \mathrm{C}$. Then, the reaction mixture was heated at the indicated temperature until completion of the reaction (TLC monitoring). According to our results, formation of AzbisPs occurs both in the presence of N-Boc and N-Cbz protecting groups, with an enhanced reactivity of the latter (Table 2, comparison between substrates $\mathbf{2 b}$ and $\mathbf{2 c}, \mathbf{2}$ and $\mathbf{2 m}$ ). Three substrates did not lead to the expected derivatives. For the bicyclic compounds $\mathbf{2} \mathbf{l}$ and $\mathbf{2 m}$, the lack of reactivity is mostly associated with the highly constrained conformation of the aziridine-ring, and the reduced product $\mathbf{3 l}$ is isolated in high yields. For the bi-aryl aziridine $\mathbf{2 n}$, the starting material is almost entirely recovered even after 4 days at reflux. Thus, the high steric hindrance of the heterocycle appears to prevent the formation of the AzbisP derivative.

Ring-opened products are observed as byproducts for a few aziridines (2f, $\mathbf{2 h}$ and $\mathbf{2 i}$ ), highlighting the competition between the formation of aziridine $\alpha$-methylene-gem-bisphosphonates and $\beta$ - 
aminophosphonates in the presence of electron-withdrawing substituent, which enhances the electrophilicity of the aziridine ring. In the case of aryl or alkyl substituents, no byproducts are isolated.

As expected, we obtained better yields at higher temperatures (from $\mathrm{rt}$ to $55^{\circ} \mathrm{C}$ ) for derivatives $\mathbf{3 b}$ and $\mathbf{3 f}$, whereas some aziridines (2a, $\mathbf{2} \mathbf{c}$ and $\mathbf{2 k}$ ) appeared unstable upon heating. Temperature has also an effect on the competition between ring-opening and AzbisP formation (no byproduct is isolated from $2 \mathrm{f}$ after refluxing the reaction mixture at $80^{\circ} \mathrm{C}$, whereas the ring-opened product is recovered, in $5 \%$ and $16 \%$ yield, respectively at room temperature and $50^{\circ} \mathrm{C}$ ) indicating that the AzbisP is the thermodynamic product of the reaction. In addition, the studied reaction does not lead to the epimerization of the aziridine ring, since compound $\mathbf{3 g}$ is not isolated as a mixture of diastereoisomers.

Table 2. Substrate scope for the formation of aziridine $\alpha$-methylene-gem-bisphosphonates

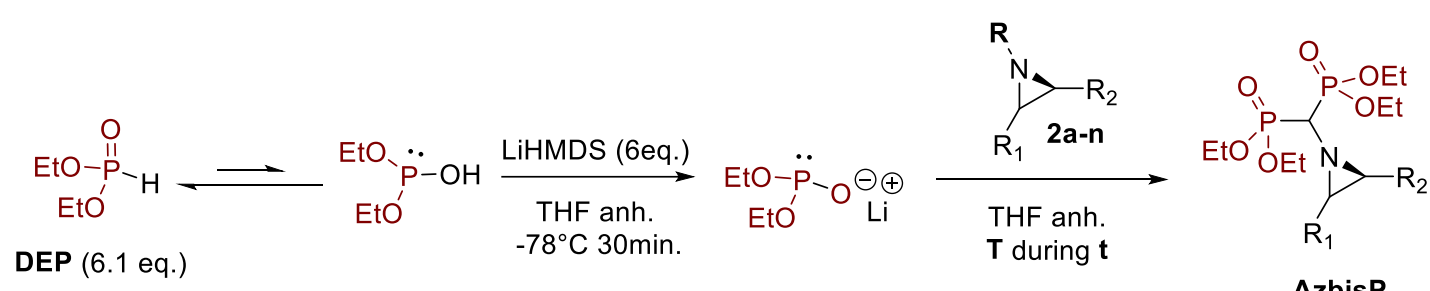

DEP (6.1 eq.)
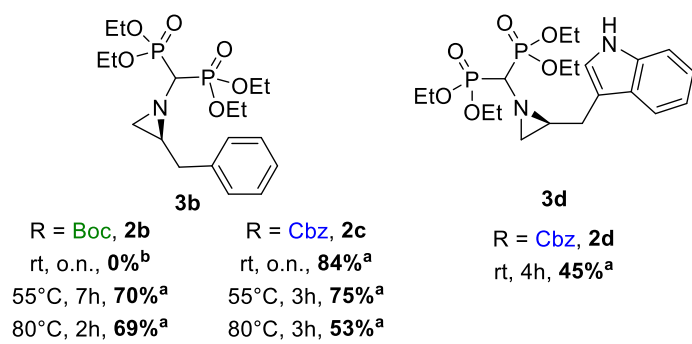

3d

$\mathrm{R}=\mathrm{Cbz}, \mathbf{2 d}$

rt, $4 \mathrm{~h}, \mathbf{4 5 \%}{ }^{\mathrm{a}}$

$55^{\circ} \mathrm{C}, 3 \mathrm{~h}, \mathbf{7 5} \%^{\mathrm{a}}$
$80^{\circ} \mathrm{C}, 3 \mathrm{~h}, \mathbf{5 3} \%^{\mathrm{a}}$

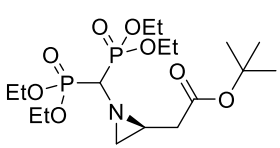

3h

$\mathbf{3 g}$
$\mathrm{R}=\mathrm{Boc}, \mathbf{2 g}$

$\mathrm{R}=\mathrm{Cbz}, \mathbf{2 h}$

rt, $2 \mathrm{~h}, 20 \%$ a,c

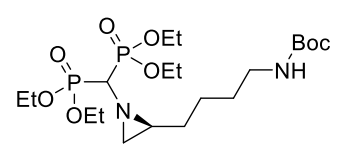

$\begin{array}{cr}\text { R = Cbz, 2i } & \text { R = Boc, 2j } \\ \text { rt, 4h, 40\% }{ }^{\mathrm{a}, \mathrm{c}} & \text { rt, o.n., 0\% } \\ & 40^{\circ} \mathrm{C}, \mathbf{4 d},<\mathbf{1 0} \%\end{array}$

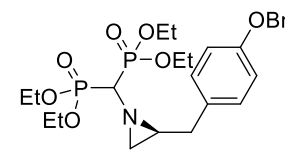

$3 \mathrm{e}$

$\mathrm{R}=\mathrm{Boc}, \mathbf{2 e}$

$80^{\circ} \mathrm{C}, 3 \mathrm{~h}, 46 \%^{\mathrm{a}}$

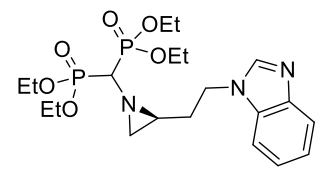

$\mathrm{R}=\mathrm{Boc}, \mathbf{2 f}$

it, o.n., $30 \% \mathrm{a}$,c

$55^{\circ} \mathrm{C}, 7 \mathrm{~h}, \mathbf{5 0} \% \mathrm{a}, \mathrm{c}$

$80^{\circ} \mathrm{C}, 3 \mathrm{~h}, 46 \%^{\mathrm{a}}$

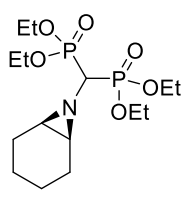

3k

$\mathrm{R}=\mathrm{Cbz}, \mathbf{2} \mathbf{k}$

rt, $2 \mathrm{~h}, 72 \%^{\mathrm{a}}$

$80^{\circ} \mathrm{C}, 80 \mathrm{~min}, 14 \%$ a

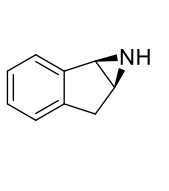

31

$\mathrm{R}=\mathrm{Cbz}, \mathbf{2 1}$

$80^{\circ} \mathrm{C}, 2 \mathrm{~h}, 97 \%^{\mathrm{a}}$

$\mathrm{R}=\mathrm{Boc}, \mathbf{2 m}$

$80^{\circ} \mathrm{C}, 2 \mathrm{~h}, 84 \%^{\mathrm{a}}$

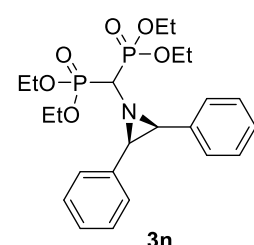

$\mathrm{R}=\mathrm{Cbz}, \mathbf{2 n}$

rt, o.n., $0 \%^{\mathrm{b}}$ $80^{\circ} \mathrm{C}, 4 \mathrm{~d}, 0 \%^{\mathrm{b}}$

Isolated yield after purification by column chromatography. Abbreviation o.n. means overnight. [a] Total conversion indicated by TLC. [b] Starting material recovered. [c] Corresponding ring-opened byproduct was also isolated (from $\mathbf{2 f}, 5 \%$ at $\mathrm{rt}$ and $16 \%$ at $55^{\circ} \mathrm{C}$ ) or detected (from $\mathbf{2 h}$ and $\mathbf{2 i}$ ).

The reaction does not occur on N-Boc protected primary amines (Table 2, side chain of the lysine derivatives $\mathbf{2} \mathbf{i}$ and $\mathbf{2} \mathbf{j}$ ). This difference of reactivity may be explained by the $\mathrm{pKaH}$ values of aziridine and aliphatic amine (7-8 vs 9-11 in $\mathrm{H}_{2} \mathrm{O}$, respectively), which has an impact on the electrophilicity of 
the carbonyl moiety. The N-carbamoyl aziridine moiety is more reactive in our conditions, thus allowing the selective formation of the AzbisP, even in the presence of another carbamate group. To validate this hypothesis, we examined the behavior of few analogues of the $N$-carbamoyl aziridine scaffold with higher $\mathrm{pKaH}$ values, such as $\mathrm{N}$-Cbz-azetidine (10-11) $\mathrm{N}$-Cbz-pyrrolidine (10.8) and $\mathrm{N}$ Cbz-piperidine (10) derivatives ( $\mathrm{pKaH}$ values have been estimated using the MarvinSketch software), ${ }^{33}$ as well as a well-known rearrangement product of $\mathrm{N}$-carbamoyl aziridines in the presence of azaphilic Lewis acids, the oxazolidine-2-one 4 (Scheme 2). ${ }^{34}$ In all cases, the pKaH of the nitrogen is higher than the one of the aziridine scaffold, and no reactivity is observed in our conditions (DEP/LiHMDS).
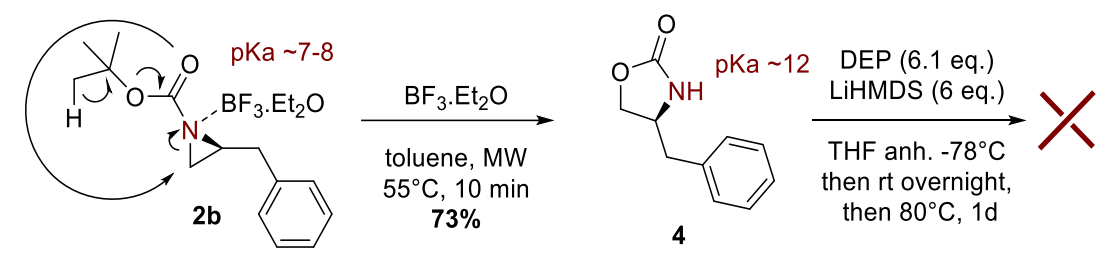

Scheme 2. Synthesis of the oxazolidine-2-one $\mathbf{4}$ from $\mathbf{2 b}$ using previously published procedure ${ }^{34}$ and its lack of reactivity towards nucleophilic attack of diethylphosphite. The chelation model and the proposed mechanism for the ring expansion of N-Boc-aziridines into oxazolines catalyzed by azaphilic Lewis acids is well-known in the literature. ${ }^{35,36}$

The structures of bisphosphonate esters (compounds $\mathbf{3 a - b}, \mathbf{3 d - i}$ and $\mathbf{3 k}$ ) were readily identified by analysis of the NMR data, including bidimensional techniques, as well as mass spectrometry, and compared to their corresponding $\mathrm{N}$-carbamoyl aziridines used as starting materials (see SI). In all cases, ${ }^{1} \mathrm{H}$ NMR spectra shows a triplet in the range of 2.4 to $2.8 \mathrm{ppm}$ with a coupling constant value of $\sim 18 \mathrm{~Hz}$ and corresponding to a single proton. The 2D spectral data $\left(\mathrm{COSY}{ }^{1} \mathrm{H} /{ }^{1} \mathrm{H}\right.$ and HSQC $1 \mathrm{H} / 13 \mathrm{C}$ ) demonstrate that this proton is not coupling with other protons of the molecules and is associated with a carbon atom, which signal is also a triplet at $\sim 65 \mathrm{ppm}$ with a wide coupling constant of $\sim 150 \mathrm{~Hz}$. This observation is consistent with the proposed structure including two phosphonate groups attached to the same carbon $(\mathrm{P}-\mathrm{CH}-\mathrm{P})$. As example, detailed study of 1D and 2D RMN spectra (see SI) for two AzbisPs (compounds 3d and 3g) and their corresponding N-carbamoyl-aziridines (compounds $\mathbf{2 d}$ and $\mathbf{2 g}$ ) highlights the characteristic signals of these compounds and peak attribution. The aziridine structure is clearly recognizable by the presence of 2 doublets at $\sim 2 \mathrm{ppm}$ in ${ }^{1} \mathrm{H}$ NMR (corresponding to the methylene of the aziridine ring) in the AzbisPs, which are also present in ${ }^{1} \mathrm{H}$ NMR spectrum of the starting material, supporting that the ring is kept intact at the end of the reaction. In addition, COSY and ${ }^{13} \mathrm{C}$ spectra also demonstrate the presence of the aziridine ring, the 2 doublets 
in ${ }^{1} \mathrm{H}$ NMR are associated to the same carbon according to HSQC experiment. The signal of the methylene protons is coupling only with the methyne proton of the aziridine ring in the COSY experiment.

Then, we get interested in deciphering the mechanism of the reaction. Because the aziridine $\mathbf{2 g}$ does not show any epimerization, we exclude the ring opening of the aziridine and its subsequent ring closing. Thus, our first hypothesis consisted in the nucleophilic attack of one equivalent of diethylphosphite anion on the carbonyl group, leading to the elimination of the alkoxide and the formation of an aziridine $\mathrm{N}$-carbamoyl phosphonate (scheme 3A). Differences of reactivity between $\mathrm{N}-\mathrm{Boc}$ and $\mathrm{N}-\mathrm{Cbz}$ substrates support this assumption as the benzyloxide ion may be considered as a better leaving group than the tert-butyloxide one. These kind of addition/elimination occurring on a carbamate moiety has already been reported in the literature. ${ }^{37}$ In addition, an acyl derivative of the 2benzyl-aziridine treated in our conditions (data not shown) did not lead to the formation of the corresponding AzbisP, which indicated the key role of the carbamoyl moiety.

This addition/elimination step may be competing with the nucleophilic attack on the aziridine ring that is also an electrophilic center. Here, we assumed that the chelation of the lithium with the ester moiety enhanced the reactivity of the carbamate, since lithium is considered as a better Lewis acid than the other alkali ions (sodium and potassium). Corresponding models and rules are presented in Scheme 3A. Herein, we proposed a different site of chelation for the Lewis acid (than the one drawn in scheme 2) on the basis of the work of T. Lectka et al., ${ }^{36}$ indicating that coordination to the carbonyl may be better at activating the substrate toward external nucleophilic attack.

In a second step, the $N$-carbamoyl phosphonate intermediate may be subject to a second nucleophilic attack of the diethylphosphite anion affording an $\alpha$-hydroxybisphosphonate derivative as a second intermediate (Scheme 3B). This scaffold is known to undergo [1,2]-phospha-Brook rearrangement (phosphonate-phosphate rearrangement) in the presence of a base, leading to the formation of a phosphate moiety (Scheme $3 \mathrm{C}) .{ }^{38-44}$ 

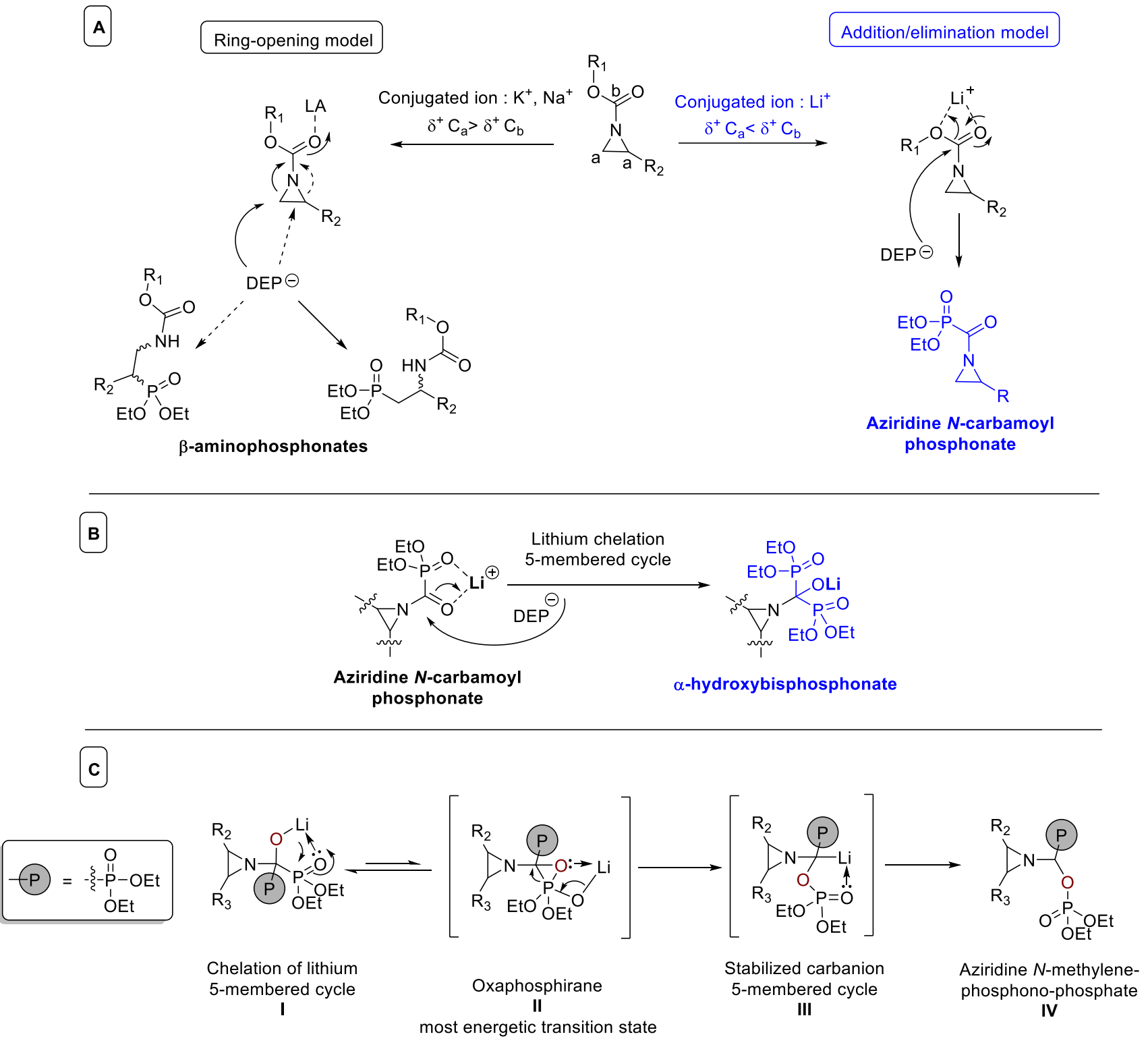

Scheme 3. Mechanistic investigation. A) Models for the ring opening of $N$-carbamates compared with the addition of DEP on the carbamate moiety in the presence of lithium cations. B) Second nucleophilic attack leading to a hypothetic $\alpha$-hydroxybisphosphonate intermediate. C) [1,2]-Phospha-Brook rearrangement with lithiated base starting from $N$-carbamoyl phosphonate aziridine.

The proposed mechanism (Scheme 3C) is adapted from the work of Ranga et al. ${ }^{45}$ when studying the $n$-BuLi mediated [1,2]-phospha-Brook rearrangement and the addition of lithiated diethylphosphite on acetophenone. Their conclusions (supported by DFT calculations and ${ }^{31} \mathrm{P}$ NMR experiments) proved the crucial role of lithium in the stabilization of the transition states. This rearrangement occurs right after the formation of the intermediate I (Scheme 3C). The lithium, conjugated to the hydroxylate, chelates the phosphonate and induces the addition of the hydroxyl anion on the phosphorus atom, 
leading to the formation of the oxaphosphirane high-energy intermediate II. The cleavage of the P-C bond of the lithium-chelated oxaphosphirane and the subsequent creation of the P-O bond forms then the 5-membered carbanion containing cycle intermediate III. The rearrangement presumably proceeds through lithium-proton exchange (involving the diethylphosphite as supposed by Ranga et al.).

To go further in the study of the impact of the lithium ion in the proposed mechanism and rearrangement as well as the nature of the base, we envisaged additional experiments (Table 3). Thus, various bases susceptible to deprotonate the diethylphosphite were tested using aziridine $\mathbf{2 f}$ as substrate. In the presence of $t \mathrm{BuOK}$ (entry 1), only 3\% of AzbisP is recovered and we mainly observed the formation of ring-opened product (47\% yield). When using NaHMDS (entry 2) or LiHMDS (entry 3) with similar pKa values, no AzbisP is observed for NaHMDS and only $9 \%$ of the ring-opened product is recovered. These results are in agreement with previous data from the literature, comparing the effect of various [M] HMDS bases on the double addition of DEP on acid chlorides, ${ }^{39}$ and the recent mechanistic study of the role of the lithium ion in the [1,2]-phospha-Brook rearrangement. ${ }^{45}$ When a stronger base such as $\mathrm{NaH}$ was used (entry 4), only traces of ring-opened product were observed.

Table 3. Influence of the nature of the base on the formation of AzbisPs.

\begin{tabular}{ccccc}
\hline \multirow{2}{*}{ Entry } & Base (6 eq.) & pKa & \multicolumn{2}{c}{ Yield (\%) } \\
\cline { 4 - 5 } 1 & $t$ BuOK & 17 & $3 \%$ & AzbisP 3f \\
2 & NaHMDS & 30 & $0 \%$ & $9 \%$ \\
3 & LiHMDS & 30 & $\mathbf{3 0 \%}$ & $5 \%$ \\
4 & $\mathrm{NaH}$ & 35 & $0 \%$ & $5 \%$ \\
\hline
\end{tabular}

Accordingly, the lithium is conjugated to the hydroxylate anion generated after the second nucleophilic attack of DEP on the acylphosphonate moiety (scheme 3C). Lithium acts as a Lewis acid as well as a conjugated cation. It is supposed to chelate the phosphonate group $\mathbf{I}$, to facilitate the formation of the oxaphosphirane II, and to stabilize the carbanion III with the formation of a strong ion pair. The proposed model (Scheme 3B) is hypothesized from the mechanism of the reverse reaction, the phosphate-phosphonate rearrangement, in the presence of strong lithiated base ${ }^{46,47}$ and on the basis of the work of Ranga et al. ${ }^{45}$ This point further reinforces the crucial role of lithium in the stabilization of the transition states. The [1,2]-phospha-Brook rearrangement likely ends by the lithium-proton exchange between DEP and the transition state III. 
In the last step, we assumed that the newly formed phosphate group may be substituted by a third equivalent of deprotonated diethylphosphite, still with the assistance of the lithium as a Lewis acid, which chelate the phosphate group and leading to the AzbisP product (Scheme 4). A similar substitution reaction is observed by Fitch and Moedritzer on a phosphonylphosphate compound, in the presence of $\mathrm{PCl}_{5}{ }^{48}$ It has to be specified that the diethylphosphoester moiety (in red, intermediate IV, Scheme 4), as well as the phosphonate group, certainly increase the electrophilicity of the vicinal carbon atom (in green, intermediate IV, Scheme 4) assisting consequently the nucleophilic attack of DEP (in blue, Scheme 4) on this position. As for the previous steps, the aziridine ring is supposed to participate in the same way because of the low pKa value of its nitrogen atom. This last step is thought to be fast, in order to consume quickly the rearranged phosphorylated compound and thus preventing the reverse reaction.

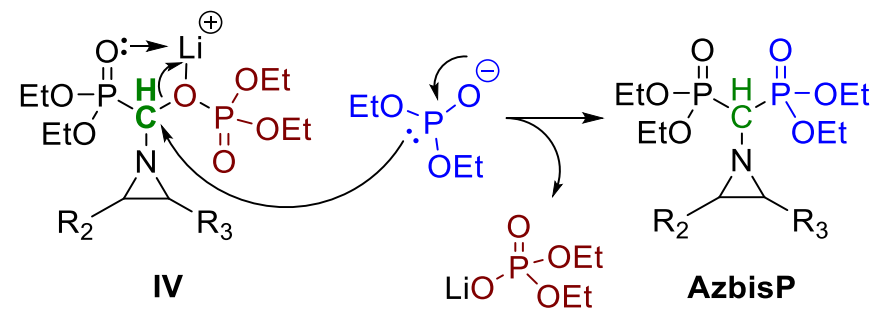

Scheme 4. Substitution of the phosphate group by a third nucleophilic attack of DEP.

Furthermore, several attempts to isolate intermediates during the reaction and to synthesize the $\mathrm{N}$ carbamoyl phosphonate intermediates were unsuccessful, thus demonstrating both high reactivity and instability of these species. When using lower equivalents of DEP/base (Table 4), total consumption of the starting $\mathrm{N}$-carbamoyl aziridine is observed but the yields of the recovered AzbisP remains below the one obtained with 6 equivalents of DEP/LiHMDS, and yet no intermediates is isolated. The similar yields obtained for 2 and 3 equivalents of the reactants (Table 4, Entry 1 and 2) may suggest that the reaction requires at least 3 equivalents of DEP/LiHMDS to be in stoichiometric conditions.

Table 4. Influence of the amounts of DEP/LiHMDS on the yield of the reaction.

\begin{tabular}{ccccc}
\hline Entry & $\begin{array}{c}\text { DEP } \\
\text { (eq.) }\end{array}$ & $\begin{array}{c}\text { LiHMDS } \\
(\text { eq. })\end{array}$ & t (h) & $\begin{array}{c}\text { Yield 3b } \\
(\%)\end{array}$ \\
\hline 1 & $\mathbf{2 . 1}$ & 2 & 6 & $\mathbf{1 3}$ \\
2 & $\mathbf{3 . 1}$ & 3 & 4.5 & $\mathbf{1 4}$ \\
3 & 4.1 & 4 & 4 & 29
\end{tabular}




\begin{tabular}{ccccc}
4 & 5.1 & 5 & 2.5 & 32 \\
5 & 6.1 & 6 & 2 & 69 \\
\hline
\end{tabular}

\section{CONCLUSION}

$\mathrm{N}$-carbamoyl aziridines are efficiently converted into their corresponding methylene-gembisphosphonylated derivatives in the presence of LiHMDS and DEP. To our knowledge, it is the first time that such reaction is reported in the literature. This transformation preferably occurs with $\mathrm{N}-\mathrm{Cbz}$ aziridines, and competes with the aziridine ring-opening reaction. A broad range of $\mathrm{N}-\mathrm{Cbz}$ and $\mathrm{N}-\mathrm{Boc}$ aziridines were synthesized to study the scope of the reaction and afforded the desired phosphonylated compounds with mild to good yield. Influence of the nature of the base, the nature of the aziridine protecting group, amount of reactants, and the aziridine substituent were studied.

According to the reaction scopes and additional experiments, we proposed a mechanism and highlighted the essentiality of the lithium ion and the basicity of the nitrogen. The latter involved a phosphonate-phosphate rearrangement newly described from a lithiated $\alpha$-hydroxy-bisphosphonate species.

\section{EXPERIMENTAL SECTION}

\section{General Information}

Reagents were from the followed suppliers: Sigma-Aldrich, Alfa Aesar, Acros or TCI. Anhydrous solvents (sealed flasks and stored on molecular sieves) were from Sigma-Aldrich (acetonitrile, $\mathrm{MeOH}$, dichloromethane, THF, DMF, $\mathrm{Et}_{2} \mathrm{O}$ ), or distillated beforehand on $\mathrm{P}_{2} \mathrm{O}_{5}$ or $\mathrm{CaCl}_{2}$ (dichloromethane), sodium and benzophenone (THF), according to protocols by Armarego et Perrin. ${ }^{49}$ Diethylphosphite (Acros) was distilled on $\mathrm{KOH}$ under reduced pressure and stored in a sealed round-bottom flask under argon atmosphere and in the dark. Reactions that required heating were performed in hot plate/heating block apparatus with internal temperature control.

Microwave conditions reactions were performed on Anton-Paar Monowave instrument and using sealed tubes, the reaction temperature was monitored by external surface sensor.

Thin-layer chromatography (TLC) was performed on precoated aluminum sheets of silica 60 F254 (Merck, Art. 5554). Visualization of products was accomplished by UV absorbance (254 nm) and/or by charring with Ninhydrin solution, «Molybden blue », or Sulfuric acid 5\% (v/v) in ethanol. Flash chromatography on silica gel were performed on the automated system Biotage ${ }^{\circledR}$ Isolera $^{\mathrm{TM}} 4$ and silica cartridges (Buchi Flashpure Silica or Biotage ${ }^{\circledR}$ ZIP Si Cartridge). 
NMR spectra were recorded in the Laboratoire de Mesures Physiques (LMP - University of Montpellier) on Brüker Avance spectrometers (400, 500 or $600 \mathrm{MHz}$ for ${ }^{1} \mathrm{H}$ NMR spectra ; 101 or 126 $\mathrm{MHz}$ for ${ }^{13} \mathrm{C}$ NMR spectra, and 162 or $202 \mathrm{MHz}$ for $\left.{ }^{31} \mathrm{P} \mathrm{NMR}\right)$, at room temperature $\left(20^{\circ} \mathrm{C}\right)$. Chemical shifts were reported in ppm (parts per million) and determined according to the solvent peak used as internal reference and relatively to the trimethylsilyl peak (TMS) for ${ }^{1} \mathrm{H}$ and ${ }^{13} \mathrm{C}$ NMR, and according to an external reference for ${ }^{31} \mathrm{P}$ NMR. Used solvents were $\mathrm{CDCl}_{3}, \mathrm{D}_{2} \mathrm{O}, \mathrm{MeOD}-\mathrm{d}_{4}$ and DSMO- $\mathrm{d}_{6}$ (Sigma-Aldrich). COSY $\left({ }^{1} \mathrm{H} 2 \mathrm{D}\right), \mathrm{HSQC}{ }^{1} \mathrm{H}_{-}{ }^{13} \mathrm{C}$, and $\mathrm{HMBC}{ }^{1} \mathrm{H}_{-}{ }^{13} \mathrm{C}$ were obtained to interpret and confirm ${ }^{1} \mathrm{H}$ and ${ }^{13} \mathrm{C}$ NMR spectra. ${ }^{1} \mathrm{H}$ NMR data are reported as follows: chemical shift (ppm), multiplicity ( $\mathrm{s}=$ singlet, $\mathrm{d}=$ doublet, $\mathrm{t}=$ triplet, $\mathrm{q}=$ quartet, $\mathrm{m}=$ multiplet, $\mathrm{br}=$ broad signal), coupling constants and integration. High-resolution mass spectra (HRMS) were recorded on a Q-TOF SynaptG2-S instrument using electrospray ionization (ESI) in positive or negative ion polarity mode.

The $\mathrm{pKa}$ and $\mathrm{pKaH}$ values calculations were performed on Marvin Sketch software (ChemAxon, version 19.13.00) ${ }^{33}$ from the compounds structures. Acidic $p K a$ value was leveled up to 50 for the calculations.

General procedure for the preparation of N-Boc and N-Cbz aziridines using the Mitsunobu reaction (Table 1 , route $A$ )

$\mathrm{N}-\mathrm{Cbz}$ or N-Boc amino-alcohol (1.0 eq.) is dissolved under argon atmosphere in anhydrous THF $(13.2 \mathrm{~mL} / \mathrm{mmol})$ at $0^{\circ} \mathrm{C}$. Triphenylphosphine (1.5 eq.) is added in one portion, followed by diethylazodicarboxylate (1.64 eq.) dropwise, at $0^{\circ} \mathrm{C}$. The reaction mixture is allowed to warm to room temperature and kept stirring until completion of the reaction (TLC monitoring). Solvents are removed in vacuum, and the crude is purified by silica gel flash chromatography (petroleum ether/ethyl acetate ) to obtain the desired compound.

\section{Benzyl (S)-2-phenylaziridine-1-carboxylate (2a)}

The compound $(S)$-2a $(1.42 \mathrm{~g}, 5.61 \mathrm{mmol}, 76 \%$ yield $)$ was obtained as an oil following the procedure described above (overnight reaction) from compound $1 \mathrm{a}(2.00 \mathrm{~g}, 7.38 \mathrm{mmol})$.

$\mathrm{R} f$ petroleum ether/EtOAc (7/3, v/v): 0.90. ${ }^{1} \mathrm{H}$ NMR (500 MHz, $\left.\mathrm{CDCl}_{3}\right) \delta 7.31-7.13$ (m, 10H, HAr), 5.07 (q, J = 12.3 Hz, 2H, $\left.\mathrm{CH}_{2} \mathrm{Ph}\right), 3.42(\mathrm{dd}, \mathrm{J}=6.3,3.6 \mathrm{~Hz}, 1 \mathrm{H}, \mathrm{CH}), 2.63\left(\mathrm{~d}, \mathrm{~J}=6.3 \mathrm{~Hz}, 1 \mathrm{H}, \mathrm{CH}_{2} \mathrm{CH}\right)$, $2.22\left(\mathrm{~d}, \mathrm{~J}=3.6 \mathrm{~Hz}, 1 \mathrm{H}, \mathrm{CH}_{2} \mathrm{CH}\right) .{ }^{13} \mathrm{C}\left\{{ }^{1} \mathrm{H}\right\} \mathrm{NMR}\left(126 \mathrm{MHz}, \mathrm{CDCl}_{3}\right) \delta 163.2(\mathrm{~s}, \mathrm{C}(\mathrm{O}) \mathrm{Cbz}), 137.0(\mathrm{~s}$, CAr), 135.8 (s, CAr), 129.0 - 127.8 (m, CHAr), 126.3 (s, CHAr), 68.5 (s, $\mathrm{CH}_{2} \mathrm{Ph}$ ), 39.5 (s, CH), 35.3 (s, $\mathrm{CH}_{2} \mathrm{CH}$ ). HRMS (ESI/Q-TOF) m/z: [M+H] ${ }^{+}$Calcd for $\mathrm{C}_{16} \mathrm{H}_{16} \mathrm{NO}_{2}$ : 254.1181; found: 254.1181 . 


\section{Benzyl (S)-2-benzylaziridine-1-carboxylate (2c)}

The compound (S)-2c (626 mg, $2.34 \mathrm{mmol}, 67 \%$ yield) was obtained as a white solid following the procedure described above (overnight stirring) from commercially available $\mathrm{N}$-Cbz-L-phenylalanilol $(1.00 \mathrm{~g}, 3.50 \mathrm{mmol})$.

$\mathrm{R} f$ hexane/EtOAc (7/3, v/v): 0.80. ${ }^{1} \mathrm{H}$ NMR (400 MHz, $\left.\mathrm{CDCl}_{3}\right) \delta 7.40-7.19(\mathrm{~m}, 10 \mathrm{H}, \mathrm{CHAr}), 5.12$ (s, $\left.2 \mathrm{H}, \mathrm{OCH}_{2} \mathrm{Ph}\right), 3.04-2.90\left(\mathrm{~m}, 1 \mathrm{H}, \mathrm{CHCH}_{2} \mathrm{Ph}\right), 2.77-2.64\left(\mathrm{~m}, 2 \mathrm{H}, \mathrm{CHAz}, \mathrm{CHCH}_{2} \mathrm{Ph}\right), 2.39$ (d, J $\left.=5.9 \mathrm{~Hz}, 1 \mathrm{H}, \mathrm{CH}_{2}, \mathrm{Az}\right), 2.10\left(\mathrm{~d}, \mathrm{~J}=3.5 \mathrm{~Hz}, 1 \mathrm{H}, \mathrm{CH}_{2}, \mathrm{Az}\right)$. Data in accordance with the literature. ${ }^{50}$

\section{Benzyl (S)-2-((1H-indol-3-yl)methyl)aziridine-1-carboxylate (2d)}

The compound $(S)$-2d (550 mg, $1.80 \mathrm{mmol}$, 49\% yield) was obtained as an oil following the procedure described above (overnight stirring) from compound $1 \mathbf{d}(1.20 \mathrm{~g}, 3.70 \mathrm{mmol})$.

$\mathrm{R} f$ petroleum ether/EtOAc (7/3, v/v): 0.43. ${ }^{1} \mathrm{H}$ NMR $\left(500 \mathrm{MHz}, \mathrm{CDCl}_{3}\right) \delta 8.00(\mathrm{~s}, 1 \mathrm{H}, \mathrm{NH}), 7.58(\mathrm{dd}$, $\mathrm{J}=7.9,0.8 \mathrm{~Hz}, 1 \mathrm{H}), 7.41-7.28$ (m, 7H, CHAr), $7.22-7.18$ (m, 1H, CHAr), 7.16 - 7.09 (m, 1H, CHAr), 5.13 (s, 2H, $\mathrm{OCH}_{2} \mathrm{Ph}$ ), 3.14 - 3.04 (m, 1H, $\mathrm{CHCH}_{2} \mathrm{C}$ ), 2.90 (ddd, J = 15.3, 5.8, $0.7 \mathrm{~Hz}, 1 \mathrm{H}$, $\left.\mathrm{CHCH}_{2} \mathrm{C}\right), 2.82(\mathrm{qd}, \mathrm{J}=5.9,3.8 \mathrm{~Hz}, 1 \mathrm{H}, \mathrm{CHAz}), 2.40\left(\mathrm{~d}, \mathrm{~J}=6.0 \mathrm{~Hz}, 1 \mathrm{H}, \mathrm{CH}_{2}, \mathrm{Az}\right), 2.14(\mathrm{~d}, \mathrm{~J}=3.7$ $\left.\mathrm{Hz}, 1 \mathrm{H}, \mathrm{CH}_{2}, \mathrm{Az}\right) .{ }^{13} \mathrm{C}\left\{{ }^{1} \mathrm{H}\right\} \mathrm{NMR}\left(126 \mathrm{MHz}, \mathrm{CDCl}_{3}\right) \delta 171.3$ (s, C(O)Boc), 163.5 (s, CAr), 136.0 (s, CAr), 129.8 - 126.6 (m, CHAr), 122.3 (d, J = 16.6 Hz, CHAr), 119.6 (s, CHAr), 118.9 (s, CHAr), 112.2 (s, CHAr), 111.3 (s, CHAr), 68.2 (s, $\mathrm{OCH}_{2} \mathrm{Ph}$ ), 38.3 (s, CHAz), 32.0 (s, $\left.\mathrm{CH}_{2}, \mathrm{Az}\right), 28.2$ (s, $\mathrm{CHCH}_{2} \mathrm{C}$ ). HRMS (ESI/Q-TOF) m/z: [M+H] $]^{+}$Calcd for $\mathrm{C}_{19} \mathrm{H}_{19} \mathrm{~N}_{2} \mathrm{O}_{2}: 307.1447$; found: 307.1446 .

\section{tert-Butyl (S)-2-(4-(benzyloxy)benzyl)aziridine-1-carboxylate (2e)}

The compound (S)-2e (766 mg, $2.26 \mathrm{mmol}, 53 \%$ yield) was obtained as an oil following the procedure described above (6h stirring) from compound $1 \mathrm{e}(1.51 \mathrm{~g}, 4.23 \mathrm{mmol})$.

$\mathrm{R} f$ petroleum ether/EtOAc (9/1, v/v): 0.42. ${ }^{1} \mathrm{H}$ NMR (500 MHz, $\left.\mathrm{CDCl}_{3}\right) \delta 7.45-7.41(\mathrm{~m}, 2 \mathrm{H}, \mathrm{CHAr})$, 7.41 - 7.35 (m, 2H, CHAr), 7.35 - 7.29 (m, 1H, CHAr), 7.24 - 7.20 (m, 2H, CHAr), 6.95 - 6.90 (m, 2H, CHAr), 5.05 (s, 2H, OCH$\left.{ }_{2} \mathrm{Ph}\right), 2.97-2.82\left(\mathrm{~m}, 1 \mathrm{H}, \mathrm{CHCH}_{2} \mathrm{Ph}\right), 2.66-2.53\left(\mathrm{~m}, 2 \mathrm{H}, \mathrm{CHCH}_{2} \mathrm{Ph}\right.$, CHAz), 2.29 (d, J = 5.9 Hz, 1H, CH $2, \mathrm{Az}), 2.01$ (d, J = 3.4 Hz, 1H, $\mathrm{CH}_{2}, \mathrm{Az}$ ), 1.44 (s, 9H, $\mathrm{CH}_{3}$, Boc). ${ }^{13} \mathrm{C}\left\{{ }^{1} \mathrm{H}\right\} \mathrm{NMR}\left(126 \mathrm{MHz}, \mathrm{CDCl}_{3}\right) \delta 162.6$ (s, C(O)Boc), 157.7 (s, CAr), 137.3 (s, CAr), $131.3-126.7$ (m, 9C, CHAr, CAr), 115.0 (s, CHAr), 81.2 (s, $\left.\underline{\mathrm{C}}\left(\mathrm{CH}_{3}\right)_{3}, \mathrm{Boc}\right), 70.2$ (s, $\left.\mathrm{OCH}_{2} \mathrm{Ph}\right), 38.7$ (s, CHAz), 37.7 (s, $\mathrm{CHCH}_{2} \mathrm{Ph}$ ), 31.5 (s, $\mathrm{CH}_{2}, \mathrm{Az}$ ), 28.1 (s, 3C, $\mathrm{CH}_{3}$, Boc). HRMS (ESI/Q-TOF) m/z: [M-H] Calcd for $\mathrm{C}_{21} \mathrm{H}_{24} \mathrm{NO}_{3}$ : 338.1756; found: 338.1753 .

\section{Benzyl (S)-2-(4-((tert-butoxycarbonyl)amino)butyl)aziridine-1-carboxylate (2i)}

The compound (S)-2i (900 mg, $2.59 \mathrm{mmol}, 95 \%$ yield) was obtained as an oil following the procedure described above (overnight stirring) from compound $\mathbf{1 i}(1.00 \mathrm{~g}, 2.73 \mathrm{mmol}$ ).

$\mathrm{R} f$ petroleum ether/EtOAc (7/3, v/v): 0.51. ${ }^{1} \mathrm{H}$ NMR (600 MHz, $\left.\mathrm{CDCl}_{3}\right) \delta 7.38-7.30$ (m, 5H, CHAr), $5.12\left(\mathrm{~s}, 2 \mathrm{H}, \mathrm{OCH}_{2} \mathrm{Ph}\right), 4.49(\mathrm{~s}, 1 \mathrm{H}, \mathrm{NH}), 3.10\left(\mathrm{~m}, 2 \mathrm{H}, \mathrm{CH}_{2} \mathrm{NH}\right), 2.46-2.38$ (m, 1H, CHAz), 2.34 (d, 
$\left.\mathrm{J}=6.1 \mathrm{~Hz}, 1 \mathrm{H}, \mathrm{CH}_{2}, \mathrm{Az}\right), 1.98\left(\mathrm{~d}, \mathrm{~J}=3.8 \mathrm{~Hz}, 1 \mathrm{H}, \mathrm{CH}_{2}, \mathrm{Az}\right), 1.55-1.45\left(\mathrm{~m}, 6 \mathrm{H}, 3 \mathrm{CH}_{2}\right), 1.44(\mathrm{~s}, 9 \mathrm{H}$, $\left.3 \mathrm{CH}_{3}, \mathrm{Boc}\right) .{ }^{13} \mathrm{C}\left\{{ }^{1} \mathrm{H}\right\} \mathrm{NMR}\left(151 \mathrm{MHz}, \mathrm{CDCl}_{3}\right) \delta 163.6$ (s, C(O)Cbz), 156.1 (s, C(O)Boc), 136.0 (s, CAr), 128.7 (s, CHAr), 128.5 (s, CHAr), 128.3 (s, CHAr), 68.2 (s, $\mathrm{OCH}_{2} \mathrm{Ph}$ ), 40.6 (s, $\mathrm{CH}_{2} \mathrm{NH}$ ), 38.3 (s, CHAz), 32.0 (s, $\mathrm{CH}_{2}, \mathrm{Az}$ ), 31.9 (s, $\mathrm{CH}_{2}$ ), 28.6 (s, 3C, 3CH 3 , Boc), 24.2 (s, 2C, $\mathrm{CH}_{2}$ ). HRMS (ESI/QTOF) m/z: [M+Na] ${ }^{+}$Calcd for $\mathrm{C}_{19} \mathrm{H}_{28} \mathrm{~N}_{2} \mathrm{O}_{4} \mathrm{Na}: 371.1947$; found: 371.1945 .

\section{Benzyl (2S,3S)-2,3-diphenylaziridine-1-carboxylate (2n)}

The compound (S)-2n (724 mg, 2.20 mmol, 76\% yield) was obtained as an oil following the procedure described above (overnight stirring) from compound $1 \mathbf{n}(1.00 \mathrm{~g}, 2.88 \mathrm{mmol})$.

Rf petroleum ether/EtOAc (7/3, v/v): 0.90. ${ }^{1} \mathrm{H}$ NMR (600 MHz, $\left.\mathrm{CDCl}_{3}\right) \delta 7.29-7.15$ (m, 13H, CHAr), $6.94(\mathrm{dd}, \mathrm{J}=7.8,1.6 \mathrm{~Hz}, 2 \mathrm{H}, \mathrm{CHAr}), 4.91$ (dd, J = 65.4, $12.1 \mathrm{~Hz}, 2 \mathrm{H}, \mathrm{OCH}_{2} \mathrm{Ph}$ ), 3.71 (s, 2H, CHAz). ${ }^{13} \mathrm{C}\left\{{ }^{1} \mathrm{H}\right\}$ NMR $\left(151 \mathrm{MHz}, \mathrm{CDCl}_{3}\right) \delta 160.5$ (s, C(O)Cbz), 135.5 (s, CAr), 129.7 - 125.5 (m, CHAr), $68.3\left(\mathrm{~s}, \mathrm{OCH}_{2} \mathrm{Ph}\right), 48.5$ (s, CHAz). HRMS (ESI/Q-TOF) m/z: $[\mathrm{M}+\mathrm{H}]^{+}$Calcd for $\mathrm{C}_{22} \mathrm{H}_{20} \mathrm{NO}_{2}$ : 330.1494; found: 330.1491 .

General Procedure for the Preparation of N-Boc and N-Cbz aziridines from amino-alcohols via onepot cyclization (Table 1 , route $B$ )

$\mathrm{N}$-Cbz or $\mathrm{N}$-Boc amino-alcohol (1 eq.) is dissolved under argon atmosphere in anhydrous diethyl ether $(15 \mathrm{~mL} / \mathrm{mmol})$ at room temperature. Dry para-toluenesulfonyl chloride (2.2 eq.) is added, followed by the addition of $\mathrm{KOH}$ grinded pellets (9.0 eq.) A drying tube filled with calcium chloride $\left(\mathrm{CaCl}_{2}\right)$ seals the condenser. The reaction mixture is heated to $40^{\circ} \mathrm{C}$ and stirred until completion of the reaction. Solvents are evaporated in vacuum, and the crude dissolved in ethyl acetate. The salts are filtered and the filtrate concentrated to obtain the desired compound. Purification step using silica gel flash chromatography (petroleum ether/ethyl acetate gradient) was only performed for compounds $\mathbf{2 b}$, $2 \mathbf{l}$ and $\mathbf{2 m}$.

\section{tert-Butyl (S)-2-benzylaziridine-1-carboxylate (2b)}

The compound (S)-2b (1.39 g, $5.96 \mathrm{mmol}, 75 \%$ yield) was obtained as a pale orange oil following the procedure described above (overnight stirring) from commercially available $N$-Boc-L-phenylalanilol $(2.00 \mathrm{~g}, 7.96 \mathrm{mmol})$.

$\mathrm{R} f$ petroleum ether/EtOAc (9/1, v/v): 0.75. ${ }^{1} \mathrm{H} \mathrm{NMR}\left(500 \mathrm{MHz}, \mathrm{CDCl}_{3}\right) \delta 7.33-7.29(\mathrm{~m}, 4 \mathrm{H}, \mathrm{CHAr})$, $7.23(\mathrm{dt}, \mathrm{J}=5.5,4.2 \mathrm{~Hz}, 1 \mathrm{H}, \mathrm{CHAr}), 2.96\left(\mathrm{dd}, \mathrm{J}=14.0,5.4 \mathrm{~Hz}, 1 \mathrm{H}, \mathrm{CH}_{2} \mathrm{Ph}\right), 2.66$ (dd, J = 18.9, 4.8 $\mathrm{Hz}, 1 \mathrm{H}, \mathrm{CH}_{2} \mathrm{Ph}$ ), 2.66 - 2.60 (m, 1H, CHAz), 2.31 (d, J = 5.7 Hz, 1H, $\left.\mathrm{CH}_{2}, \mathrm{Az}\right), 2.03$ (d, J = 3.5 Hz, $\left.1 \mathrm{H}, \mathrm{CH}_{2}, \mathrm{Az}\right), 1.44$ (s, $9 \mathrm{H}, 3 \mathrm{CH}_{3}$, Boc). Data in accordance with the literature. ${ }^{51}$ 


\section{tert-Butyl (S)-2-((S)-sec-butyl)aziridine-1-carboxylate (2g)}

The compound (S)-2g (891 mg, $4.47 \mathrm{mmol}, 97 \%$ yield) was obtained as an oil following the procedure described above (overnight stirring) from commercially available $N$-Boc-L-isoleucinol (1.00 g, 4.60 mmol).

$\mathrm{R} f$ hexane/EtOAc (7/3, v/v): 0.85. ${ }^{1} \mathrm{H}$ NMR $\left(600 \mathrm{MHz}, \mathrm{CDCl}_{3}\right) \delta 2.22(\mathrm{~d}, \mathrm{~J}=6.3 \mathrm{~Hz}, 1 \mathrm{H}, \mathrm{CH}, \mathrm{Az})$, $2.18-2.14$ (m, 1H, CHAz), 1.90 (d, J = 3.9 Hz, 1H, $\left.\mathrm{CH}_{2}, \mathrm{Az}\right), 1.65$ (tdd, J = 14.5, 7.3, 5.0 Hz, 1H, $\left.\mathrm{CH}_{2} \mathrm{CH}_{3}\right), 1.44$ (s, 9H, 3CH 3 , Boc), $1.41-1.32\left(\mathrm{~m}, 1 \mathrm{H}, \mathrm{CH}_{2} \mathrm{CH}_{3}\right), 1.17-1.09$ (m, 1H, $\left.\mathrm{CHCH}_{3}\right), 0.97$ $\left(\mathrm{t}, \mathrm{J}=7.5 \mathrm{~Hz}, 3 \mathrm{H}, \mathrm{CH}_{3} \mathrm{CH}_{2}\right), 0.91\left(\mathrm{~d}, \mathrm{~J}=6.8 \mathrm{~Hz}, 3 \mathrm{H}, \mathrm{CH}_{3} \mathrm{CH}\right) .{ }^{13} \mathrm{C}\left\{{ }^{1} \mathrm{H}\right\} \mathrm{NMR}\left(151 \mathrm{MHz}, \mathrm{CDCl}_{3}\right) \delta$ 163.0 (s, C(O)Boc), 80.9 (s, $\left.\mathrm{C}\left(\mathrm{CH}_{3}\right)_{3}, \mathrm{Boc}\right), 43.5$ (s, $\left.\mathrm{CHAz}\right), 37.8\left(\mathrm{~s}, \mathrm{CHCH}_{3}\right), 30.7$ (s, $\left.\mathrm{CH}_{2}, \mathrm{Az}\right), 28.1$ (s, 3C, 3CH 3 , Boc), 27.7 (s, $\left.\mathrm{CH}_{2} \mathrm{CH}_{3}\right), 16.1$ (s, $\left.\mathrm{CH}_{3} \mathrm{CH}\right), 11.1$ (s, $\mathrm{CH}_{3} \mathrm{CH}_{2}$ ). HRMS (ESI/Q-TOF) m/z: $[\mathrm{M}+\mathrm{H}]^{+}$Calcd for $\mathrm{C}_{11} \mathrm{H}_{22} \mathrm{NO}_{2}$ : 200.1645; found: 200.1643 .

\section{tert-Butyl (S)-2-(4-((tert-butoxycarbonyl)amino)butyl)aziridine-1-carboxylate (2j)}

The compound (S)-2j (284 mg, $0.90 \mathrm{mmol}$, quantitative yield) was obtained as an following the procedure described above (4h stirring) from compound $\mathbf{1 j}$ (300 mg, $0.90 \mathrm{mmol}$ ).

$\mathrm{R} f$ Dichloromethane/MeOH (95/5, v/v): 0.70. ${ }^{1} \mathrm{H}$ NMR (400 MHz, $\left.\mathrm{CDCl}_{3}\right) \delta 4.55$ (s, 1H, NH), 3.12 (dd, J = 9.0, 4.2 Hz, 2H, $\mathrm{CH}_{2} \mathrm{NH}$ ), 2.34 (dt, J = 9.8, 4.9 Hz, 1H, CHAz), 2.25 (d, J = 6.1 Hz, 1H, $\mathrm{CH}_{2}$, $\mathrm{Az}), 1.90\left(\mathrm{~d}, \mathrm{~J}=3.8 \mathrm{~Hz}, 1 \mathrm{H}, \mathrm{CH}_{2}, \mathrm{Az}\right), 1.53\left(\mathrm{~m}, 6 \mathrm{H}, 3 \mathrm{CH}_{2}\right), 1.45$ (s, 9H, $\left.\mathrm{CH}_{3}, \mathrm{Boc}\right), 1.44$ (s, 9H, $\mathrm{CH}_{3}$, Boc). ${ }^{13} \mathrm{C}\left\{{ }^{1} \mathrm{H}\right\}$ NMR (101 MHz, $\left.\mathrm{CDCl}_{3}\right) \delta 162.8$ (s, C(O),Boc Az), 156.1 (s, C(O)Boc), 81.1 (s, $\left.\mathrm{C}\left(\mathrm{CH}_{3}\right)_{3}, \mathrm{Boc}\right), 79.2$ (s, $\mathrm{C}\left(\mathrm{CH}_{3}\right)_{3}$, Boc), $40.6\left(\mathrm{~s}, \mathrm{CH}_{2} \mathrm{NH}\right), 38.0$ (s, $\left.\mathrm{CHAz}\right), 32.0\left(\mathrm{~s}, \mathrm{CH}_{2}\right), 31.8\left(\mathrm{~s}, \mathrm{CH}_{2}\right.$,

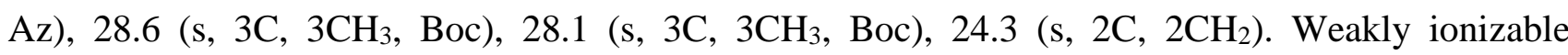
compound, molecular peak undetected in MS analysis, neither in positive or negative mode.

\section{Benzyl (1R,6S)-7-azabicyclo[4.1.0]heptane-7-carboxylate (2k)}

The compound $(S)-2 k$ (789 mg, $3.41 \mathrm{mmol}$, 97\% yield) was obtained as a pale orange oil following the procedure described above (5h stirring) from compound $1 \mathbf{k}(1 \mathrm{~g}, 3.5 \mathrm{mmol})$.

$\mathrm{R} f$ petroleum ether/EtOAc (9/1, v/v): 0.50. ${ }^{1} \mathrm{H} \mathrm{NMR}\left(400 \mathrm{MHz}, \mathrm{CDCl}_{3}\right) \delta 7.40-7.28(\mathrm{~m}, 5 \mathrm{H}, \mathrm{CHAr})$, $5.12\left(\mathrm{~s}, 2 \mathrm{H}, \mathrm{CH}_{2} \mathrm{Ph}\right), 2.71-2.59(\mathrm{~m}, 2 \mathrm{H}, 2 \mathrm{CHAz}), 2.03-1.72\left(\mathrm{~m}, 4 \mathrm{H}, 2 \mathrm{CH}_{2}\right), 1.46-1.20(\mathrm{~m}, 4 \mathrm{H}$, $\left.2 \mathrm{CH}_{2}\right)$. Data in accordance with the literature. ${ }^{32}$ Weakly ionizable compound, molecular peak undetected in MS analysis, neither in positive or negative mode.

\section{Benzyl (1aR,6aS)-6,6a-dihydroindeno[1,2-b]azirine-1(1aH)-carboxylate (invertomers) (2I)}

The compound $(S)$-2l (150 mg, $0.57 \mathrm{mmol}, 16 \%$ yield) was obtained as an oil following the procedure described above (overnight stirring) from compound 11 (1.00 g, $3.53 \mathrm{mmol}$ ).

$\mathrm{R} f$ hexane/EtOAc (7/3, v/v): 0.30. ${ }^{1} \mathrm{H}$ NMR (400 MHz, $\left.\mathrm{CDCl}_{3}\right) \delta 7.43-7.31$ (m, 8H, CHAr, A,B), $7.30-7.22$ (m, 5H, CHAr, A,B), 5.15 (s, 0.68H, $\left.\mathrm{CH}_{2} \mathrm{Ph}, \mathrm{A}\right), 5.13$ (s, 2H, $\left.\mathrm{CH}_{2} \mathrm{Ph}, \mathrm{B}\right), 5.10$ (d, J = 2.9 
$\mathrm{Hz}, 0.42 \mathrm{H}, \mathrm{CCHAz}, \mathrm{A}), 5.05$ (d, J = 5.2 Hz, 1H, CCHAz, B), 4.45 (t, J = 9.5 Hz, 1H, $\mathrm{CHAzCH}_{2}, \mathrm{~B}$ ), $4.06\left(\mathrm{t}, \mathrm{J}=6.7 \mathrm{~Hz}, 0.12 \mathrm{H}, \mathrm{CHAzCH}_{2}, \mathrm{~A}\right), 3.34\left(\mathrm{dd}, \mathrm{J}=15.3,8.2 \mathrm{~Hz}, 0.33 \mathrm{H}, \mathrm{CH}_{2}, \mathrm{~A}\right), 3.26(\mathrm{dd}, \mathrm{J}=$ 15.8, 7.2 Hz, 1H, $\left.\mathrm{CH}_{2}, \mathrm{~B}\right), 2.90\left(\mathrm{dd}, \mathrm{J}=15.8,7.3 \mathrm{~Hz}, 1 \mathrm{H}, \mathrm{CH}_{2}, \mathrm{~B}\right), 2.72(\mathrm{dd}, \mathrm{J}=15.3,9.1 \mathrm{~Hz}, 0.29 \mathrm{H}$, $\mathrm{CH}_{2}$, A). HRMS (ESI/Q-TOF) m/z: [M+H] ${ }^{+}$Calcd for $\mathrm{C}_{17} \mathrm{H}_{16} \mathrm{NO}_{2}$ : 266.1176; found: 266.1162 .

\section{tert-Butyl (1aR,6aS)-6,6a-dihydroindeno[1,2-b]azirine-1(1aH)-carboxylate (invertomers) (2m)}

The compound $(S)-\mathbf{2 m}$ (480 mg, $2.08 \mathrm{mmol}, 31 \%$ yield) was obtained as an oil following the procedure described above (2h stirring) from compound $1 \mathrm{~m}(1.67 \mathrm{~g}, 6.70 \mathrm{mmol})$.

$\mathrm{R} f$ hexane/EtOAc (7/3, v/v): 0.50. ${ }^{1} \mathrm{H}$ NMR $\left(600 \mathrm{MHz}, \mathrm{CDCl}_{3}\right) \delta 7.42(\mathrm{~d}, \mathrm{~J}=7.3 \mathrm{~Hz}, 2 \mathrm{H}, \mathrm{CHAr}, \mathrm{A}, \mathrm{B})$, 7.32 - 7.19 (m, 4H, CHAr, A,B), 5.06 (d, J = 6.2 Hz, 0.42H, CCHAz, A), 5.04 (d, J = 5.2 Hz, 1H, CCHAz, B), 4.37 (s, 1H, CHAzCH 2, B), 4.08 (dd, J = 15.6, 7.9 Hz, 0.37H, $\mathrm{CHAzCH}_{2}, \mathrm{~A}$ ), 3.30 (dd, J $\left.=15.2,8.2 \mathrm{~Hz}, 0.55 \mathrm{H}, \mathrm{CH}_{2}, \mathrm{~A}\right), 3.23\left(\mathrm{dd}, \mathrm{J}=15.8,7.3 \mathrm{~Hz}, 1 \mathrm{H}, \mathrm{CH}_{2}, \mathrm{~B}\right), 2.87(\mathrm{dd}, \mathrm{J}=15.8,7.2 \mathrm{~Hz}$, 1H, $\left.\mathrm{CH}_{2}, \mathrm{~B}\right), 2.69$ (dd, J = 15.2, $\left.9.1 \mathrm{~Hz}, 0.56 \mathrm{H}, \mathrm{CH}_{2}, \mathrm{~A}\right), 1.48$ (s, 5H, $\left.\mathrm{CH}_{3}, \mathrm{Boc} \mathrm{A}\right), 1.47$ (s, 9H, $\mathrm{CH}_{3}$, Boc B). ${ }^{13} \mathrm{C}\left\{{ }^{1} \mathrm{H}\right\}$ NMR (151 MHz, $\left.\mathrm{CDCl}_{3}\right) \delta 156.2$ (s, C(O)Boc), 142.3 (s, CAr), 141.2 (s, CAr), 130.8 - 123.3 (m, CHAr), 82.1 (s, CCHAz, A), 75.0 (s, CCHAz, B), 62.0 (s, $\mathrm{CH}_{2} \mathrm{CHAz}, \mathrm{A}$ ), 54.8 (s, $\mathrm{CH}_{2} \mathrm{CHAz}, \mathrm{B}$ ), 37.0 (s, $\mathrm{CH}_{2}$, B), 36.2 (s, $\mathrm{CH}_{2}, \mathrm{~A}$ ), 28.5 (s, 3C, $\mathrm{CH}_{3}$, Boc). HRMS (ESI/Q-TOF) m/z: $[\mathrm{M}+\mathrm{H}]^{+}$Calcd for $\mathrm{C}_{14} \mathrm{H}_{18} \mathrm{NO}_{2}$ : 232.1332; found: 232.1329 .

\section{Synthesis of aziridine $2 f$}

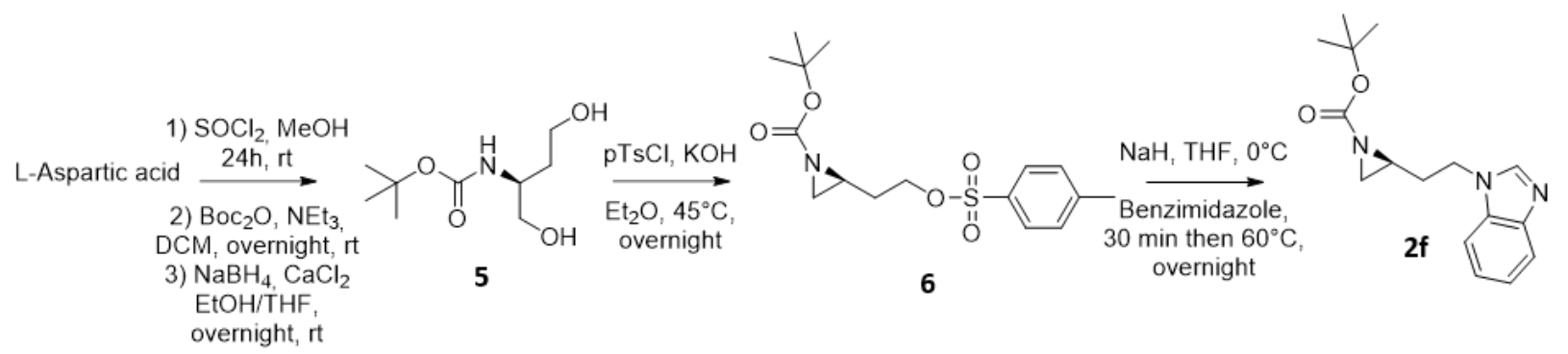

\section{tert-Butyl (S)-(1,4-dihydroxybutan-2-yl)carbamate (5)}

The protocol is adapted from Jorrës et al. ${ }^{29}$ Compound 5 was isolated as oil (6.54 g, $31.80 \mathrm{mmol}, 73 \%$ yield) from L-aspartic acid. Data in accordance with the literature. ${ }^{29}$

\section{tert-Butyl (S)-2-(2-(tosyloxy)ethyl)aziridine-1-carboxylate (6)}

The procedure is adapted from Aaseng et al. ${ }^{52}$ The compound 5 (2.96 g, $14.40 \mathrm{mmol}$, 1 eq.) was dissolved in diethyl ether $(15 \mathrm{~mL} / \mathrm{mmol})$ under argon atmosphere at room temperature. Paratoluenesulfonyl chloride (2.2 eq.) was added, followed by grinded $\mathrm{KOH}$ pellets (6 eq.). The reaction mixture was then heated at $45^{\circ} \mathrm{C}$ and kept under stirring overnight. Volatiles were removed in vacuum, and purified by silica gel flash chromatography (petroleum ether/ethyl acetate gradient) to obtain the title compound as an oil (3.15 g, $9.23 \mathrm{mmol}, 64 \%$ yield). 
R $f$ hexane/EtOAc $(6 / 4, \mathrm{v} / \mathrm{v}): 0.65 .[\alpha]_{\mathrm{D}}{ }^{20}+23^{\circ}\left(\mathrm{c} 1, \mathrm{CH}_{2} \mathrm{Cl}_{2}\right) .{ }^{1} \mathrm{H}$ NMR $\left(500 \mathrm{MHz}, \mathrm{CDCl}_{3}\right) \delta 7.80(\mathrm{~d}, \mathrm{~J}$ $=8.2 \mathrm{~Hz}, 1 \mathrm{H}, \mathrm{HAr}, \mathrm{Ts}), 7.35$ (d, J = 8.2 Hz, 1H, HAr, Ts), $4.23-4.14$ (m, 2H, $\mathrm{CH}_{2} \mathrm{O}$ ), 2.45 (s, 3H, $\mathrm{CH}_{3}, \mathrm{Ts}$ ), $2.43-2.38$ (m, 1H, CHAz), 2.26 (d, J = 6.5 Hz, 1H, $\mathrm{CH}_{2}, \mathrm{Az}$ ), 1.92 (d, J = $3.4 \mathrm{~Hz}, 1 \mathrm{H}, \mathrm{CH}_{2}$, $\mathrm{Az}), 1.90-1.84\left(\mathrm{~m}, 1 \mathrm{H}, \mathrm{CH}_{2} \mathrm{CH}_{2} \mathrm{O}\right), 1.79-1.72\left(\mathrm{~m}, 1 \mathrm{H}, \mathrm{CH}_{2} \mathrm{CH}_{2} \mathrm{O}\right), 1.42\left(\mathrm{~s}, 9 \mathrm{H}, \mathrm{CH}_{3}, \mathrm{Boc}\right) .{ }^{13} \mathrm{C}\left\{{ }^{1} \mathrm{H}\right\}$ NMR (126 MHz, $\mathrm{CDCl}_{3}$ ) $\delta 161.1$ (s, NC(O)O, Boc Az), 143.9 (s, $\left.\mathrm{CCH}_{3}, \mathrm{Ts}\right), 132.0$ (s, CS, Ts), 128.9 (m, 2C, CH, Ts), 126.9 (m, 2C, CH, Ts), 80.4 (s, $\mathrm{CCH}_{3}, \mathrm{Boc}$ ), 67.0 (s, $\mathrm{CH}_{2} \mathrm{O}$ ), 33.3 (s, CHAz), 30.9 (s, $\mathrm{CH}_{2} \mathrm{CH}_{2} \mathrm{~N}$ ), 30.4 (s, $\mathrm{CH}_{2}, \mathrm{Az}$ ), 26.8 (s, 3C, $\mathrm{CH}_{3}, \mathrm{Boc}$ ), 20.6 (s, $\mathrm{CH}_{3}$, Ts). HRMS (ESI/Q-TOF) m/z: $[\mathrm{M}+\mathrm{Na}]^{+}$Calcd for $\mathrm{C}_{16} \mathrm{H}_{24} \mathrm{NO}_{5} \mathrm{SNa}$ : 364.1195; found: 364.1194 .

\section{(S)-tert-Butyl 2-(2-(1H-benzimidazol-1-yl)ethyl)aziridine-1-carboxylate (2f)}

$\mathrm{NaH}$ (256 mg, 60\% in oil, 1.2 eq.) was dissolved in anhydrous THF (4.76 mL/mmol of benzimidazole) at $0^{\circ} \mathrm{C}$, under argon atmosphere. Benzimidazole $(760 \mathrm{mg}, 6.44 \mathrm{mmol}, 1.2 \mathrm{eq}$.$) previously dissolved in$ anhydrous THF $(2.38 \mathrm{~mL} / \mathrm{mmol}$ of benzimidazole) was added and the mixture was stirred for 30 minutes. The mixture was warmed to room temperature and the $N$-Boc tosylated aziridine 6 ( $1.83 \mathrm{~g}$, $5.36 \mathrm{mmol}, 1$ eq.) dissolved in anhydrous THF ( $3 \mathrm{~mL} / \mathrm{mmol}$ of aziridine) was added. The mixture was then heated to $60^{\circ} \mathrm{C}$ and kept under stirring overnight. Volatiles were removed under vacuum and the crude was dissolved in ethyl acetate. After filtration (glass filter, porosity 4), the salts were washed with ethyl acetate and the filtrates were combined and concentrated, then purified by flash chromatography on silica gel (petroleum ether/ethyl acetate gradient) to obtain the title compound as a colorless oil (750 mg, $2.61 \mathrm{mmol}, 50 \%$ yield). Thus, compound $2 \mathbf{f}$ was obtained in 5 steps with $22 \%$ overall yield from L-aspartic acid.

Rf EtOAc: 0.30. ${ }^{1} \mathrm{H}$ NMR (500 MHz, $\left.\mathrm{CDCl}_{3}\right) \delta 8.06$ (s, 1H, NCHArN), $7.81(\mathrm{dd}, \mathrm{J}=6.7,1.9 \mathrm{~Hz}, 1 \mathrm{H}$, HAr), 7.43 (dd, J = 7.0, 2.1 Hz, 1HAr), $7.34-7.27$ (m, 2H, HAr), 4.41 (dd, J = 7.8, $5.8 \mathrm{~Hz}, 2 \mathrm{H}$, $\mathrm{CH}_{2} \mathrm{CH}_{2} \mathrm{~N}$ ), $2.36(\mathrm{ddt}, \mathrm{J}=8.3,6.2,3.8 \mathrm{~Hz}, 1 \mathrm{H}, \mathrm{CHN}), 2.29\left(\mathrm{~d}, \mathrm{~J}=6.2 \mathrm{~Hz}, 1 \mathrm{H}, \mathrm{CHCH}_{2} \mathrm{~N}\right), 2.27-2.20$ $\left(\mathrm{m}, 1 \mathrm{H}, \mathrm{CH}_{2} \mathrm{CH}_{2} \mathrm{~N}\right), 1.91\left(\mathrm{~d}, \mathrm{~J}=3.6 \mathrm{~Hz}, 1 \mathrm{H}, \mathrm{CHCH}_{2} \mathrm{~N}, \mathrm{Az}\right), 1.72(\mathrm{ddt}, \mathrm{J}=14.3,8.5,5.8 \mathrm{~Hz}, 1 \mathrm{H}$, $\mathrm{CH}_{2} \mathrm{CH}_{2} \mathrm{~N}$ ), 1.48 (s, 9H, 3CH 3 , Boc). ${ }^{13} \mathrm{C}\left\{{ }^{1} \mathrm{H}\right\} \mathrm{NMR}\left(126 \mathrm{MHz}, \mathrm{CDCl}_{3}\right) \delta 162.22$ (s, C(O), Boc), 144.2 (s, CAr), 143.5 (s, C-2), 133.7 (s, CAr), 123.1 (s, CAr), 122.3 (s, CAr), 120.7 (s, CAr), 109.7 (s, CAr), 81.9 (s, $\left.\mathrm{C}\left(\mathrm{CH}_{3}\right)_{3}, \mathrm{Boc}\right), 43.1$ (s, $\left.\mathrm{CH}_{2} \mathrm{CH}_{2} \mathrm{~N}\right), 35.2$ (s, $\left.\mathrm{CHN}\right), 32.8\left(\mathrm{~s}, \mathrm{CH}_{2} \mathrm{CH}_{2} \mathrm{~N}\right), 31.8\left(\mathrm{~s}, \mathrm{CHCH}_{2} \mathrm{~N}\right)$, 28.1 (s, 3C, $3 \mathrm{CH}_{3}$, Boc). HRMS (ESI/Q-TOF) m/z: [M+H] ${ }^{+}$Calcd for $\mathrm{C}_{16} \mathrm{H}_{22} \mathrm{~N}_{3} \mathrm{O}_{2}$ : 288.1712; found: 288.1718 .

\section{Benzyl (R)-2-(2-(tert-butoxy)-2-oxoethyl)aziridine-1-carboxylate (2h)}

The procedure is adapted from Jung et al. ${ }^{30} \mathrm{Cbz}-\mathrm{L}-\mathrm{Asp}(\mathrm{OtBu})-\mathrm{OH}(882 \mathrm{mg}, 2.73 \mathrm{mmol}$, 1 eq.) was dissolved in anhydrous THF $(3.2 \mathrm{~mL} / \mathrm{mmol})$ under argon atmosphere, at $0^{\circ} \mathrm{C}$. $\mathrm{BH}_{3}$. THF $(1 \mathrm{M}$ in THF, 5 eq.) was added portion wise. The mixture was stirred for $1 \mathrm{~h}$ at $0^{\circ} \mathrm{C}$ then allowed to warm at room 
temperature and quenched by slow addition of methanol. The mixture is concentrated in vacuum. The crude was purified on silica gel by flash chromatography (dichloromethane/ethyl acetate gradient) to obtain the amino-alcohol ${ }^{30}$ as oil (508 mg, $1.64 \mathrm{mmol}, 60 \%$ yield). For the next step, the protocol from Aaseng et al. ${ }^{31}$ was adapted. The reduced compound (508 mg, $1.64 \mathrm{mmol}, 1$ eq.) was dissolved under argon atmosphere in anhydrous THF $(13.2 \mathrm{~mL} / \mathrm{mmol})$ à $0^{\circ} \mathrm{C}$. Triphenylphosphine (1.5 eq.) was added, followed by diethylazodicarboxylate $\left(1.64\right.$ eq.) dropwise, at $0^{\circ} \mathrm{C}$. The mixture was allowed to warm to room temperature for $2.5 \mathrm{~h}$. Solvents were evaporated in vacuum and the crude was purified by silica gel flash chromatography (petroleum ether/ethyl acetate gradient) to obtain the desired compound (296 $\mathrm{mg}, 1.02 \mathrm{mmol}, 62 \%$ yield) as an oil. Compound $\mathbf{2 h}$ was obtained in 2 steps with $37 \%$ overall yield from Cbz-L-Asp(OtBu)-OH.

$\mathrm{R} f$ petroleum ether/EtOAc (6/4, v/v): 0.90. ${ }^{1} \mathrm{H}$ NMR (400 MHz, $\left.\mathrm{CDCl}_{3}\right) \delta 7.37$ - $7.26(\mathrm{~m}, 5 \mathrm{H}, \mathrm{CHAr})$, 5.16 - 5.09 (m, 2H, $\left.\mathrm{CH}_{2} \mathrm{Ph}\right), 2.79$ (ddd, J = 12.6, 6.0, 3.7 Hz, 1H, CHAz), 2.60 (dd, J = 16.1, $5.9 \mathrm{~Hz}$, $\left.1 \mathrm{H}, \mathrm{CHCH}_{2} \mathrm{C}(\mathrm{O})\right), 2.42\left(\mathrm{~d}, \mathrm{~J}=6.1 \mathrm{~Hz}, 1 \mathrm{H}, \mathrm{CH}_{2}, \mathrm{Az}\right), 2.28\left(\mathrm{dd}, \mathrm{J}=16.1,6.7 \mathrm{~Hz}, 1 \mathrm{H}, \mathrm{CHCH}_{2} \mathrm{C}(\mathrm{O})\right.$ ), $2.07\left(\mathrm{~d}, \mathrm{~J}=3.7 \mathrm{~Hz}, 1 \mathrm{H}, \mathrm{CH}_{2}, \mathrm{Az}\right), 1.44\left(\mathrm{~s}, 9 \mathrm{H}, 3 \mathrm{CH}_{3}, \mathrm{Boc}\right)$. Data in accordance with the literature. ${ }^{53}$

\section{(S)-4-Benzyloxazolidin-2-one (4)}

The protocol is adapted from Cardillo et al. ${ }^{34}$ The compound $2 \mathbf{b}$ (160 $\mathrm{mg}, 0.68 \mathrm{mmol}$, 1 eq.) was dissolved in anhydrous toluene $(35.7 \mathrm{~mL} / \mathrm{mmol})$, at room temperature under argon atmosphere, in a microwave tube. $\mathrm{BF}_{3} \cdot \mathrm{Et}_{2} \mathrm{O}$ (1 eq.) was added, and then the tube was sealed and placed under microwave radiations $\left(55^{\circ} \mathrm{C}\right.$ for $10 \mathrm{~min}, 100 \mathrm{~W}$, and $\left.1000 \mathrm{rpm}\right)$. The reaction mixture was diluted with ethyl acetate and water. The aqueous layer was extracted twice with ethyl acetate, and the organic layers were combined, dried on $\mathrm{MgSO}_{4}$, then filtered, concentrated in vacuum, and the crude was purified by silica gel flash chromatography (dichloromethane/MeOH gradient) to afford the title compound ( $88 \mathrm{mg}, 0.49 \mathrm{mmol}, 73 \%$ yield) as a white solid.

$\mathrm{R} f$ dichloromethane/MeOH (95/5, v/v): 0.30. ${ }^{1} \mathrm{H}$ NMR $\left(500 \mathrm{MHz}, \mathrm{CDCl}_{3}\right) \delta 7.42-7.13(\mathrm{~m}, 5 \mathrm{H}$, CHAr), $5.38(\mathrm{~d}, \mathrm{~J}=4.0 \mathrm{~Hz}, 1 \mathrm{H}, \mathrm{NH}), 4.87(\mathrm{dq}, \mathrm{J}=8.0,6.7 \mathrm{~Hz}, 1 \mathrm{H}, \mathrm{CH}), 3.58(\mathrm{t}, \mathrm{J}=8.4 \mathrm{~Hz}, 1 \mathrm{H}$, $\left.\mathrm{OCH}_{2}\right), 3.34\left(\mathrm{dd}, \mathrm{J}=8.5,6.9 \mathrm{~Hz}, 1 \mathrm{H}, \mathrm{OCH}_{2}\right), 3.15\left(\mathrm{dd}, \mathrm{J}=14.0,6.2 \mathrm{~Hz}, 1 \mathrm{H}, \mathrm{CH}_{2} \mathrm{Ph}\right), 2.95(\mathrm{dd}, \mathrm{J}=$ 14.0, $\left.6.8 \mathrm{~Hz}, 1 \mathrm{H}, \mathrm{CH}_{2} \mathrm{Ph}\right) .{ }^{13} \mathrm{C}\left\{{ }^{1} \mathrm{H}\right\} \mathrm{NMR}\left(126 \mathrm{MHz}, \mathrm{CDCl}_{3}\right) \delta 159.7$ (s, C(O)), 135.3 (s, CAr), 129.5 (s, CHAr), 128.9 (s, CHAr), 127.3 (s, CHAr), 77.2 (s, CH), 45.1 (s, $\mathrm{OCH}_{2}$ ), 40.7 (s, $\left.\mathrm{CH}_{2} \mathrm{Ph}\right) . \mathrm{HRMS}$ (ESI/Q-TOF) m/z: [M+H] $]^{+}$Calcd for $\mathrm{C}_{10} \mathrm{H}_{12} \mathrm{NO}_{2}$ : 178.0868; found: 178.0868 . 


\section{Benzyl (S)-(2-hydroxy-1-phenylethyl)carbamate (1a)}

The title compound (3.76 g, $13.87 \mathrm{mmol}$, 95\% yield) was obtained as a white powder following the procedure described by Sultane et al. ${ }^{54}$ from the commercially available L-phenylglycinol $(2.00 \mathrm{~g}$, $14.58 \mathrm{mmol})$.

Rf petroleum ether/EtOAc (6/4, v/v): 0.26. ${ }^{1} \mathrm{H}$ NMR (400 MHz, $\left.\mathrm{CDCl}_{3}\right) \delta 7.42-7.23\left(\mathrm{~m}, 10 \mathrm{H}, \mathrm{CH}_{\mathrm{Ar}}\right)$, 5.47 (s, 1H, NH), 5.11 (q, J = $\left.12.2 \mathrm{~Hz}, 2 \mathrm{H}, \mathrm{OCH}_{2} \mathrm{Ph}\right), 4.86(\mathrm{~s}, 1 \mathrm{H}, \mathrm{CH}), 3.95-3.80\left(\mathrm{~m}, 2 \mathrm{H}, \mathrm{CH}_{2} \mathrm{OH}\right)$. Data in accordance with the literature. ${ }^{54}$

\section{Benzyl (S)-(1-hydroxy-3-(1H-indol-3-yl)propan-2-yl)carbamate (1d)}

The title compound (720 mg, $2.22 \mathrm{mmol}, 42 \%$ yield) was obtained as a white foam following the procedure from Yeung et al., ${ }^{55}$ from the commercially available L-tryptophanol (1.00 g, $\left.5.26 \mathrm{mmol}\right)$. $\mathrm{R} f$ petroleum ether/EtOAc (6/4, v/v): 0.10. ${ }^{1} \mathrm{H}$ NMR (400 MHz, $\left.\mathrm{CDCl}_{3}\right) \delta 8.03(\mathrm{~s}, 1 \mathrm{H}, \mathrm{NH}), 7.65(\mathrm{~d}, \mathrm{~J}$ $=7.5 \mathrm{~Hz}, 1 \mathrm{H}, \mathrm{CHAr}), 7.43-7.28(\mathrm{~m}, 6 \mathrm{H}), 7.21$ (t, J = 7.5 Hz, 1H, CHAr), 7.15 - 7.07 (m, 1H, CHAr), 7.03 (s, 1H, CHAr), 5.10 (s, 2H, $\mathrm{CH}_{2} \mathrm{Ph}$ ), 5.04 (br s, 1H, NH), $4.16-3.95$ (m, 1H, CH), 3.68 (ddd, J $\left.=15.4,9.7,4.2 \mathrm{~Hz}, 2 \mathrm{H}, \mathrm{CCH}_{2} \mathrm{CH}\right), 3.03\left(\mathrm{~d}, \mathrm{~J}=6.8 \mathrm{~Hz}, 2 \mathrm{H}, \mathrm{CH}_{2} \mathrm{OH}\right)$. Data in accordance with the literature. ${ }^{55,56}$

\section{tert-Butyl (S)-(1-(4-(benzyloxy)phenyl)-3-hydroxypropan-2-yl)carbamate (1e)}

The protocol was adapted from Jung et $a .^{30}$ Boc-Tyr(OBn)-OH (1.00 g, $2.69 \mathrm{mmol}$, 1 eq.) was dissolved in anhydrous THF $(2.3 \mathrm{~mL} / \mathrm{mmol})$ at $0^{\circ} \mathrm{C}$ under argon atmosphere. $\mathrm{BH}_{3}$.THF $(1 \mathrm{M}$ in THF, 2.5 eq.) was added dropwise over 20 minutes (exothermic reaction) to the solution and the reaction mixture was stirred $1 \mathrm{~h}$ at $0^{\circ} \mathrm{C}$, then $3 \mathrm{~h}$ at room temperature. Methanol was slowly added at $0^{\circ} \mathrm{C}$ to quench the reaction. Volatiles were removed in vacuum and the crude was dissolved in methanol then concentrated several times. The crude was purified by flash chromatography on silica gel (dichloromethane/ $\mathrm{MeOH}$ gradient) to obtain the title product as a white solid (958 $\mathrm{mg}, 2.68 \mathrm{mmol}$, 99\% yield).

$\mathrm{R} f$ Dichloromethane/MeOH (95/5, v/v): 0.55. ${ }^{1} \mathrm{H} \mathrm{NMR}\left(400 \mathrm{MHz}, \mathrm{CDCl}_{3}\right) \delta 7.47-7.29(\mathrm{~m}, 5 \mathrm{H}$, CHAr), 7.12 (d, J = 8.5 Hz, 2H, CHAr), 6.92 (d, J = 8.5 Hz, 2H, CHAr), 5.05 (s, 2H, $\mathrm{CCH}_{2} \mathrm{Ph}$ ), 3.82 (m, 1H, CH), 3.60 (ddd, J = 43.3, 11.3, 5.4 Hz, 2H, $\left.\mathrm{CHCH}_{2} \mathrm{Ph}\right), 2.78$ (d, J = 7.2 Hz, 2H, CH $\left.2 \mathrm{OH}\right), 1.42$ $\left(\mathrm{s}, 9 \mathrm{H}, 3 \mathrm{CH}_{3}\right.$, Boc). Data in accordance with the literature. ${ }^{30,57}$

\section{Benzyl tert-butyl (6-hydroxyhexane-1,5-diyl)(S)-dicarbamate (1i)}

The L-Na-Z,Ne-BocLys-OH (2.00 g, $5.26 \mathrm{mmol}, 1$ eq.) was dissolved in anhydrous THF (11.0 $\mathrm{mL} / \mathrm{mmol}$ ) under argon atmosphere. $N$-methylmorpholine and ethyl chloroformate (1 eq.) were added 
at $-10^{\circ} \mathrm{C}$ and the reaction mixture was stirred for 30 minutes at $-10^{\circ} \mathrm{C}$. $\mathrm{LiAlH}_{4}$ (2 eq.) was then slowly added dropwise and the reaction was allowed to warm at room temperature. The reaction mixture was stirred overnight at room temperature. The reaction was quenched by the slow addition of a $4.5 \%$ $\mathrm{NaOH}$ solution in water, at $0^{\circ} \mathrm{C}$. After stirring for $1 \mathrm{~h} 30$, the reaction mixture was filtered on a glass filter with dichloromethane. The filtrate was concentrated, dissolved in ethyl acetate and washed with water. Organic layer was dried over $\mathrm{MgSO}_{4}$, filtered and concentrated in vacuum. The resulting crude was purified by silica gel flash chromatography (dichloromethane/ $\mathrm{MeOH}$ gradient) to obtain the title compound as an oil (1.53 g, $4.18 \mathrm{mmol}, 80 \%$ yield $)$.

$\mathrm{R} f$ dichloromethane/MeOH (95/5, v/v): 0.40. ${ }^{1} \mathrm{H}$ NMR (400 MHz, $\left.\mathrm{CDCl}_{3}\right) \delta 7.40-7.28(\mathrm{~m}, 5 \mathrm{H}$, CHAr), 5.10 (s, 2H, $\left.\mathrm{CH}_{2} \mathrm{Ph}\right), 5.15-5.01(\mathrm{~m}, 3 \mathrm{H}), 4.57$ (s, 1H), $3.74-3.54(\mathrm{~m}, 3 \mathrm{H}), 3.22-3.13$ (m, $1 \mathrm{H}), 3.12-3.01(\mathrm{~m}, 1 \mathrm{H}), 2.46($ br s, $1 \mathrm{H}, \mathrm{OH}), 1.67-1.57(\mathrm{~m}, 2 \mathrm{H}), 1.53-1.33(\mathrm{~m}, 17 \mathrm{H})$. Data in accordance with the literature. ${ }^{58}$

\section{Di-tert-butyl (6-hydroxyhexane-1,5-diyl)(S)-dicarbamate (1j)}

The L-N $\alpha, N \varepsilon$-bisBoc-Lys-OH dicyclohexylammonium (600 mg, 1.14 mmol, 1 eq.) was dissolved in anhydrous THF $(11 \mathrm{~mL} / \mathrm{mmol})$ under argon atmosphere. $N$-methylmorpholine and ethyl chloroformate (1 eq.) were added at $-10^{\circ} \mathrm{C}$ and the reaction mixture was stirred for 30 minutes at $-10^{\circ} \mathrm{C}$. $\mathrm{LiAlH}_{4}(2.0$ eq.) was then slowly added dropwise and the reaction was allowed to warm at room temperature. The reaction mixture was stirred overnight and then quenched by the slow addition of a $4.5 \% \mathrm{NaOH}$ solution in water, at $0^{\circ} \mathrm{C}$. After stirring for $1 \mathrm{~h} 30$, the reaction mixture was filtered on a glass filter. The filtrate was concentrated in vacuum, dissolved in ethyl acetate and washed with water. Organic layer was dried over $\mathrm{MgSO}_{4}$, filtered and concentrated in vacuum. The crude was purified by silica gel flash chromatography (dichloromethane/MeOH gradient) to obtain the title compound as a colorless oil (300 $\mathrm{mg}, 0.90 \mathrm{mmol}, 80 \%$ yield).

$\mathrm{R} f$ dichloromethane/MeOH (95/5, v/v): 0.25. ${ }^{1} \mathrm{H} \mathrm{NMR}\left(400 \mathrm{MHz}, \mathrm{CDCl}_{3}\right) \delta 4.77$ (br s, $\left.1 \mathrm{H}, \mathrm{NH}\right), 4.58$ (br s, 1H, NH), $3.72-3.46\left(\mathrm{~m}, 3 \mathrm{H}, \mathrm{CH}_{2}, \mathrm{CH}\right), 3.23-3.13(\mathrm{~m}, 1 \mathrm{H}), 3.13-3.05(\mathrm{~m}, 1 \mathrm{H}), 2.69-2.43(\mathrm{~m}$, $1 \mathrm{H}, \mathrm{OH}), 1.63-1.54\left(\mathrm{~m}, 2 \mathrm{H}, \mathrm{CH}_{2}\right), 1.52-1.25\left(\mathrm{~m}, 22 \mathrm{H}, 6 \mathrm{CH}_{3}, \mathrm{Boc}, 2 \mathrm{CH}_{2}\right)$. Data in accordance with the literature. ${ }^{59}$

\section{Benzyl ((1R,2R)-2-hydroxycyclohexyl)carbamate (1k)}

The protocol was adapted from Luna et al. ${ }^{60}$ The $(1 R, 2 R)$-trans-2-aminocyclohexanol hydrochloride ( $2.00 \mathrm{~g}, 13.19 \mathrm{mmol}, 1$ eq.) was dissolved in distilled water $(1.7 \mathrm{~mL} / \mathrm{mmol})$ and $\mathrm{K}_{2} \mathrm{CO}_{3}(1.2$ eq. $)$ was added. Then benzyl chloroformate $\left(\mathrm{CbzCl}, 1.2\right.$ eq.) was added dropwise at $0^{\circ} \mathrm{C}$. A white precipitate was formed. The reaction mixture was allowed to warm to room temperature and stirred overnight. 
Dichloromethane was added to the reaction mixture and the aqueous layer was extracted with dichloromethane. Organic layers were combined, dried on $\mathrm{MgSO}_{4}$, filtered and concentrated in vacuum. The crude was purified on silica gel (petroleum ether/ethyl acetate gradient) to afford the title compound (3.72 g, $13.00 \mathrm{mmol}, 98 \%$ yield) as a white solid.

$\mathrm{R} f$ petroleum ether/EtOAc (7/3, v/v): 0.20. ${ }^{1} \mathrm{H}$ NMR (400 MHz, $\left.\mathrm{CDCl}_{3}\right) \delta 7.40-7.28(\mathrm{~m}, 5 \mathrm{H}, \mathrm{CHAr})$, $5.20-5.04\left(\mathrm{~m}, 2 \mathrm{H}, \mathrm{CH}_{2} \mathrm{Ph}\right), 4.72$ (br s, 1H, NH), $3.40(\mathrm{~m}, 1 \mathrm{H}, \mathrm{CH}), 3.32(\mathrm{~m}, 1 \mathrm{H}, \mathrm{CH}), 2.80$ (br s, 1H, $\mathrm{OH}), 2.11-1.93\left(\mathrm{~m}, 2 \mathrm{H}, \mathrm{CH}_{2}\right), 1.71\left(\mathrm{~m}, 2 \mathrm{H}, \mathrm{CH}_{2}\right), 1.40-1.09(\mathrm{~m}, 4 \mathrm{H})$. Data in accordance with the literature. ${ }^{60}$

\section{Benzyl ((1R,2R)-2-hydroxy-2,3-dihydro-1H-inden-1-yl)carbamate (11)}

The (1R,2R)-(-)-1-amino-2-indanol (1.00 g, $6.70 \mathrm{mmol}, 1 \mathrm{eq}$.) was dissolved in anhydrous dichloromethane $(1 \mathrm{~mL} / \mathrm{mmol})$ at $0^{\circ} \mathrm{C}$ under argon atmosphere. Benzyl chloroformate ( $\mathrm{CbzCl}, 1$ eq.) was added at $0^{\circ} \mathrm{C}$, followed by pyridine $(1.5$ eq. $)$, and the reaction mixture was stirred at room temperature overnight. The reaction was quenched by addition of brine and water was added to dissolve the salts. The mixture was extracted with dichloromethane and the resulting emulsion was filtered on a hydrophobic filter cartridge. Organic layers were recovered, dried on $\mathrm{MgSO}_{4}$, filtered and concentrated in vacuum. The crude was purified by silica gel flash chromatography (petroleum ether/ethyl acetate gradient) to obtain the title compound (1.20 g, $4.24 \mathrm{mmol}, 63 \%$ yield) as a white solid.

$\mathrm{R} f$ hexane/EtOAc (66/34, v/v): 0.30. ${ }^{1} \mathrm{H}$ NMR (600 MHz, $\left.\mathrm{CDCl}_{3}\right) \delta 7.39(\mathrm{dd}, \mathrm{J}=4.2,1.8 \mathrm{~Hz}, 2 \mathrm{H}$, CHAr), 7.38 - 7.33 (m, 1H, CHAr), 7.29 - 7.17 (m, 6H, CHAr), 5.23 (s, 1H, NH), 5.19 (q, J = 12.2 $\left.\mathrm{Hz}, 1 \mathrm{H}, \mathrm{OCH}_{2} \mathrm{Ph}\right), 4.99$ (t, J = 6.1 Hz, 1H, CHNH), $4.46(\mathrm{dd}, \mathrm{J}=14.7,7.4 \mathrm{~Hz}, 1 \mathrm{H}, \mathrm{CHOH}), 3.31(\mathrm{dd}$, $\left.\mathrm{J}=15.8,7.7 \mathrm{~Hz}, 1 \mathrm{H}, \mathrm{CH}_{2}\right), 2.93\left(\mathrm{dd}, \mathrm{J}=15.8,8.1 \mathrm{~Hz}, 1 \mathrm{H}, \mathrm{CH}_{2}\right) .{ }^{13} \mathrm{C}\left\{{ }^{1} \mathrm{H}\right\} \mathrm{NMR}\left(151 \mathrm{MHz}, \mathrm{CDCl}_{3}\right) \delta$ 157.9 (s, C(O)Cbz), 140.3 (s, CAr), 139.1 (s, CAr), 136.1 (s, CAr), 128.7 (m, CHAr), 127.5 (s, CHAr), 125.4 (s, CHAr), 123.1 (s, CHAr), 82.2 (s, $\mathrm{CHOH}), 67.6$ (s, $\left.\mathrm{OCH}_{2} \mathrm{Ph}\right), 64.6$ (s, 1C, CHNH), 38.6 (s, $\mathrm{CH}_{2}$ ). HRMS (ESI/Q-TOF) m/z: [M+H] $]^{+}$Calcd for $\mathrm{C}_{17} \mathrm{H}_{18} \mathrm{NO}_{3}$ : 284.1281; found: 284.1285.

\section{tert-Butyl ((1R,2R)-2-hydroxy-2,3-dihydro-1H-inden-1-yl)carbamate (1m)}

The (1R,2R)-(-)-1-amino-2-indanol (1.00 g, $6.70 \mathrm{mmol}, 1 \mathrm{eq}$.$) was dissolved in anhydrous$ dichloromethane $(1.8 \mathrm{~mL} / \mathrm{mmol})$ at room temperature under argon atmosphere. Triethylamine $\left(\mathrm{Et}_{3} \mathrm{~N}\right.$, 1.5 eq.) was added, followed by $\mathrm{Boc}_{2} \mathrm{O}$ (di-tert-butyl dicarbonate, 1.2 eq.). The reaction was stirred at room temperature for $6 \mathrm{~h}$. Reaction was quenched by addition of an aqueous solution of saturated $\mathrm{NaHCO}_{3}$ and extracted with dichloromethane. The emulsion was filtered on a hydrophobic filter 
cartridge. The organic layer was dried over $\mathrm{MgSO}_{4}$, filtered and concentrated in vacuum to obtain the title compound (1.67 g, $6.7 \mathrm{mmol}$, quantitative yield) as a white solid.

R $f$ Hexane/EtOAc (66/34, v/v): 0.50. ${ }^{1} \mathrm{H}$ NMR (600 MHz, $\left.\mathrm{CDCl}_{3}\right) \delta 7.30-7.14$ (m, 4H, CHAr), 5.06 (s, 1H, NH), 4.90 (t, J = 5.9 Hz, 1H, CHNH), 4.41 (dd, J = 14.2, $7.8 \mathrm{~Hz}, 1 \mathrm{H}, \mathrm{CHOH}), 4.30$ (s, 1H, $\mathrm{OH}), 3.28\left(\mathrm{dd}, \mathrm{J}=15.8,7.7 \mathrm{~Hz}, 1 \mathrm{H}, \mathrm{CH}_{2}\right), 2.91\left(\mathrm{dd}, \mathrm{J}=15.7,8.1 \mathrm{~Hz}, 1 \mathrm{H}, \mathrm{CH}_{2}\right), 1.50\left(\mathrm{~s}, 9 \mathrm{H}, \mathrm{CH}_{3}\right.$, Boc). ${ }^{13} \mathrm{C}\left\{{ }^{1} \mathrm{H}\right\}$ NMR (151 MHz, $\left.\mathrm{CDCl}_{3}\right) \delta 157.6$ (s, C(O)Boc), 140.3 (s, $\mathrm{CArCH}_{2}$ ), 139.5 (s, CArCHNH), 128.6 (s, CH), 127.3 (s, CH), 125.3 (s, CH), 123.1 (s, CH), 82.2 (s, CHOH), 80.6 (s, $\left.\mathrm{C}\left(\mathrm{CH}_{3}\right)_{3}\right), 64.2(\mathrm{~s}, \mathrm{CHNH}), 38.5\left(\mathrm{~s}, \mathrm{CH}_{2}\right), 28.6$ (s, $\mathrm{CH}_{3}$, Boc). HRMS (ESI/Q-TOF) m/z: $[\mathrm{M}+\mathrm{H}]^{+} \mathrm{Calcd}$ for $\mathrm{C}_{14} \mathrm{H}_{20} \mathrm{NO}_{3}$ : 250.1437; found: 250.1434.

\section{Benzyl ((1S,2R)-2-hydroxy-1,2-diphenylethyl)carbamate (1n)}

The (1R,2S)-2-amino-1,2-diphenylethanol (2.00 g, $9.38 \mathrm{mmol}, 1$ eq.) was dissolved in anhydrous THF $(2.77 \mathrm{~mL} / \mathrm{mmol})$ at $0^{\circ} \mathrm{C}$ under argon atmosphere. Solid $\mathrm{NaHCO}_{3}$ (2 eq.) was then added, followed by benzyl chloroformate $(\mathrm{CbzCl}, 1.2$ eq.) dropwise. The mixture was stirred overnight at room temperature until completion of the reaction (TLC monitoring). The reaction was quenched by addition of water and extracted with ethyl acetate. Organic layers were acidified with $\mathrm{HCl} 1 \mathrm{~N}$, washed with brine, dried on $\mathrm{MgSO}_{4}$, filtrated and concentrated in vacuum. The crude was purified by silica gel flash chromatography (dichloromethane/MeOH gradient) to obtain the title compound (3.25 g, $9.38 \mathrm{mmol}$, quantitative yield) as a white powder.

$\mathrm{R} f$ petroleum ether/EtOAc (7/3, v/v): 0.50. ${ }^{1} \mathrm{H}$ NMR (400 MHz, $\left.\mathrm{CDCl}_{3}\right) \delta 7.41-7.28$ (m, 5H, CHAr), $7.24-7.19$ (m, 6H, CHAr), 7.04 (m, 4H, CHAr), 5.59 (br s, 1H, NH), $5.15-4.99$ (m, 4H, CHOH, $\mathrm{CHNH}, \mathrm{CH}_{2} \mathrm{Ph}$ ), 2.39 (br s, $1 \mathrm{H}, \mathrm{OH}$ ). Data in accordance with the literature. ${ }^{61}$

General Procedure for the Preparation of N-methylene-gem-bisphosphonates aziridines from $N$ carbamoyl aziridines

Freshly distilled diethylphosphite (6.1 eq.) was dissolved in anhydrous THF $(0.5 \mathrm{~mL} / \mathrm{mmol}$ of diethylphosphite) under argon atmosphere, and cooled to $-78^{\circ} \mathrm{C}$. Then LiHMDS (1M in THF, 6 eq.) was added and the reaction mixture was stirred for 30 minutes at $-78^{\circ} \mathrm{C}$. A solution of the $N$-carbamoyl aziridine (1 eq.) in anhydrous THF $(2.8 \mathrm{~mL} / \mathrm{mmol}$ of aziridine) was added dropwise to the reaction, which was then allowed to warm to room temperature. The reaction mixture was stirred at temperature $\mathrm{T}^{\circ}$ until completion of the reaction (TLC monitoring). The reaction was quenched by addition of a saturated aqueous solution of $\mathrm{NH}_{4} \mathrm{Cl}$ until dissolution of the salts formed. The mixture was extracted twice with ethyl acetate. Organic layers were dried on $\mathrm{MgSO}_{4}$, filtered and concentrated in vacuum 
and the crude was purified by silica gel flash chromatography (dichloromethane/MeOH gradient) to afford the desired product.

\section{Tetraethyl ((2-phenylaziridin-1-yl)methylene)(S)-bis(phosphonate) (3a)}

The title compound (100 mg, $0.39 \mathrm{mmol}, 45 \%$ yield) was obtained as a colorless oil following the procedure described above, after stirring $1 \mathrm{~h}$ at room temperature, from compound $\mathbf{2 a}$ (73 $\mathrm{mg}, 0.18$ mmol).

$\mathrm{R} f$ dichloromethane/MeOH (95/5, v/v): 0.30. ${ }^{1} \mathrm{H} \mathrm{NMR}\left(400 \mathrm{MHz}, \mathrm{CDCl}_{3}\right) \delta 7.31-7.24(\mathrm{~m}, 4 \mathrm{H}$, CHAr), 7.21 (m, 1H, CHAr), $4.37-4.24\left(\mathrm{~m}, 4 \mathrm{H}, 2 \mathrm{CH}_{2} \mathrm{CH}_{3}\right), 4.21-3.98\left(\mathrm{~m}, 4 \mathrm{H}, 2 \mathrm{CH}_{2} \mathrm{CH}_{3}\right), 2.84$ (ddd, J = 6.1, 4.1, 1.7 Hz, 1H, CHAz), 2.61 (t, J = 18.0 Hz, 1H, PCHP), 2.19 (d, J = 3.9 Hz, 1H, CH 2 , $\mathrm{Az}), 2.14\left(\mathrm{dd}, \mathrm{J}=6.7,1.8 \mathrm{~Hz}, 1 \mathrm{H}, \mathrm{CH}_{2}, \mathrm{Az}\right), 1.39\left(\mathrm{td}, \mathrm{J}=7.1,4.1 \mathrm{~Hz}, 6 \mathrm{H}, 2 \mathrm{CH}_{2} \mathrm{CH}_{3}\right), 1.18(\mathrm{dt}, \mathrm{J}=$ 14.2, 7.1 Hz, 6H, 2CH $\left.\mathrm{CH}_{3}\right) .{ }^{13} \mathrm{C}\left\{{ }^{1} \mathrm{H}\right\} \mathrm{NMR}\left(126 \mathrm{MHz}, \mathrm{CDCl}_{3}\right) \delta 139.3$ (s, CAr), 128.2 (s, CHAr), 127.2 (s, CHAr), 126.5 (s, CHAr), 65.1 (t, J = 149.3 Hz, PCHP), 63.6 (d, J = 7.1 Hz, 2C, $\mathrm{CH}_{2} \mathrm{CH}_{3}$ ), $63.4-63.1\left(\mathrm{~m}, 2 \mathrm{C}, \mathrm{CH}_{2} \mathrm{CH}_{3}\right), 43.6(\mathrm{~d}, \mathrm{~J}=15.6 \mathrm{~Hz}, \mathrm{CHAz}), 40.2\left(\mathrm{~d}, \mathrm{~J}=15.9 \mathrm{~Hz}, \mathrm{CH}_{2}, \mathrm{Az}\right), 16.8-$ $16.6\left(\mathrm{~m}, 2 \mathrm{C}, 2 \mathrm{CH}_{2} \mathrm{CH}_{3}\right), 16.4\left(\mathrm{~d}, \mathrm{~J}=6.8 \mathrm{~Hz}, 2 \mathrm{C}, 2 \mathrm{CH}_{2} \mathrm{CH}_{3}\right) .{ }^{31} \mathrm{P}\left\{{ }^{1} \mathrm{H}\right\} \mathrm{NMR}\left(162 \mathrm{MHz}, \mathrm{CDCl}_{3}\right) \delta 17.6$ $\left(\mathrm{d}, \mathrm{J}_{\mathrm{PP}}=2.6 \mathrm{~Hz}\right), 17.4\left(\mathrm{~d}, \mathrm{~J}_{\mathrm{PP}}=2.6 \mathrm{~Hz}\right) . \mathrm{HRMS}(\mathrm{ESI} / \mathrm{Q}-\mathrm{TOF}) \mathrm{m} / \mathrm{z}:[\mathrm{M}+\mathrm{H}]^{+}$Calcd for $\mathrm{C}_{17} \mathrm{H}_{30} \mathrm{NO}_{6} \mathrm{P}_{2}$ : 406.1548; found: 406.1549 .

\section{Tetraethyl ((2-benzylaziridin-1-yl)methylene)(S)-bis(phosphonate) (3b)}

The title compound (264 mg, $0.63 \mathrm{mmol}, 84 \%$ yield) was obtained as a colorless oil following the procedure described above (overnight stirring at room temperature) from compound $2 \mathbf{c}$ (200 $\mathrm{mg}, 0.75$ $\mathrm{mmol}$ ) or from compound $\mathbf{2 b}$ (stirring $2 \mathrm{~h}$ at $80^{\circ} \mathrm{C}, 500 \mathrm{mg}, 2.14 \mathrm{mmol}$ ) with $69 \%$ yield $(630 \mathrm{mg}, 1.50$ mmol).

$\mathrm{R} f$ dichloromethane/MeOH (95/5, v/v): 0.35. ${ }^{1} \mathrm{H}$ NMR $\left(500 \mathrm{MHz}, \mathrm{CDCl}_{3}\right) \delta 7.30-7.18(\mathrm{~m}, 5 \mathrm{H}$, CHAr), 4.32 - $4.18\left(\mathrm{~m}, 8 \mathrm{H}, \mathrm{CH}_{2} \mathrm{CH}_{3}\right), 3.33\left(\mathrm{dd}, \mathrm{J}=14.1,3.6 \mathrm{~Hz}, 1 \mathrm{H}, \mathrm{CH}_{2} \mathrm{Ph}\right), 2.40$ (t, J = 18.1 Hz, 1H, PCHP), 2.40 (dd, J = 13.4, 9.4 Hz, 1H, $\left.\mathrm{CH}_{2} \mathrm{Ph}\right), 2.11-2.00(\mathrm{~m}, 1 \mathrm{H}, \mathrm{CHAz}), 1.93$ (d, J = 3.9 Hz, 1H, $\left.\mathrm{CH}_{2}, \mathrm{Az}\right), 1.72$ (d, J = 8.3 Hz, 1H, $\left.\mathrm{CH}_{2}, \mathrm{Az}\right), 1.38\left(\mathrm{t}, \mathrm{J}=7.1 \mathrm{~Hz}, 6 \mathrm{H}, \mathrm{CH}_{2} \mathrm{CH}_{3}\right), 1.35$ (dt, J = 7.0, $\left.4.4 \mathrm{~Hz}, 6 \mathrm{H}, \mathrm{CH}_{2} \mathrm{CH}_{3}\right) .{ }^{13} \mathrm{C}\left\{{ }^{1} \mathrm{H}\right\} \mathrm{NMR}\left(126 \mathrm{MHz} \mathrm{CDCl}_{3}\right) \delta 138.8$ (s, CAr), 129.1 (s, 2C, CHAr), 128.5 (s, 2C, CHAr), 126.4 (s, CHAr), 64.9 (t, J = 150.1 Hz, PCHP), 63.3 (d, J = $10.6 \mathrm{~Hz}, 2 \mathrm{C}, \mathrm{CH}_{2} \mathrm{CH}_{3}$ ), $63.1\left(\mathrm{~d}, \mathrm{~J}=6.9 \mathrm{~Hz}, 2 \mathrm{C}, \mathrm{CH}_{2} \mathrm{CH}_{3}\right), 43.1$ (d, J = 16.2 Hz, CHAz), 38.9 (s, $\mathrm{CH}_{2} \mathrm{Ph}$ ), 36.5 (d, J = 17.0 Hz, $\left.\mathrm{CH}_{2} \mathrm{Az}\right), 16.7\left(\mathrm{~d}, \mathrm{~J}=10.3 \mathrm{~Hz}, 2 \mathrm{C}, \mathrm{CH}_{2} \mathrm{CH}_{3}\right), 16.6\left(\mathrm{~d}, \mathrm{~J}=10.1 \mathrm{~Hz}, 2 \mathrm{C}, \mathrm{CH}_{2} \mathrm{CH}_{3}\right) .{ }^{31} \mathrm{P}\left\{{ }^{1} \mathrm{H}\right\} \mathrm{NMR}(202$ $\left.\mathrm{MHz}, \mathrm{CDCl}_{3}\right) \delta 18.0(\mathrm{~s}, 1 \mathrm{P}), 17.9$ (s, 1P). HRMS (ESI/Q-TOF) m/z: [M+H] ${ }^{+}$Calcd for $\mathrm{C}_{18} \mathrm{H}_{32} \mathrm{NO}_{6} \mathrm{P}_{2}$ : 420.1705; found: 420.1709 .

\section{Tetraethyl ((2-((1H-indol-3-yl)methyl)aziridin-1-yl)methylene)(S)-bis(phosphonate) (3d)}

The title compound (53 mg, $0.11 \mathrm{mmol}, 45 \%$ yield) was obtained as a colorless oil following the procedure described above (4h stirring at room temperature) from compound $2 \mathbf{d}$ ( $78 \mathrm{mg}, 0.25 \mathrm{mmol}$ ). 
$\mathrm{R} f$ dichloromethane/MeOH (95/5, v/v): 0.50. ${ }^{1} \mathrm{H}$ NMR (600 MHz, $\left.\mathrm{CDCl}_{3}\right) \delta 8.46(\mathrm{~s}, 1 \mathrm{H}, \mathrm{NH}), 7.60$ (dd, J = 7.8, 0.7 Hz, 1H, CHAr), 7.36 (d, J = 8.1 Hz, 1H, CHAr), 7.19 - 7.11 (m, 1H, CHAr), 7.11 7.05 (m, 1H, CHAr), 7.02 (d, J = 2.0 Hz, 1H, NHCHArC), $4.35-4.17$ (m, 8H, 4CH $\mathrm{CH}_{3}$ ), 3.50 (dd, J $\left.=14.8,3.3 \mathrm{~Hz}, 1 \mathrm{H}, \mathrm{CHCH}_{2} \mathrm{C}\right), 2.55-2.47\left(\mathrm{~m}, 1 \mathrm{H}, \mathrm{CHCH}_{2} \mathrm{C}\right), 2.43$ (t, J = 18.2 Hz, 1H, PCHP), 2.19 - 2.13 (m, 1H, CHAz), 1.96 (d, J = 4.0 Hz, 1H, CH $2, \mathrm{Az}), 1.72$ (d, J = 7.0 Hz, 1H, CH2, Az), 1.39 $1.36\left(\mathrm{~m}, 6 \mathrm{H}, 2 \mathrm{CH}_{2} \mathrm{CH}_{3}\right), 1.37-1.33\left(\mathrm{~m}, 6 \mathrm{H}, 2 \mathrm{CH}_{2} \mathrm{CH}_{3}\right) .{ }^{13} \mathrm{C}\left\{{ }^{1} \mathrm{H}\right\} \mathrm{NMR}\left(151 \mathrm{MHz}, \mathrm{CDCl}_{3}\right) \delta 136.4$ (s, CAr), 127.8 (s, CAr), 122.1 (s, CHAr), 121.9 (s, CHAr), 119.3 (s, CHAr), 119.1 (s, CHAr), 112.8 (s, CAr), 111.3 (s, CCHArNH), 65.1 (t, J = 150.3 Hz, PCHP), $63.4-62.7$ (m, 4C, $\mathrm{CH}_{2} \mathrm{CH}_{3}$ ), 42.8 (d, $\mathrm{J}=16.1 \mathrm{~Hz}, \mathrm{CHAz}), 37.0\left(\mathrm{~d}, \mathrm{~J}=16.8 \mathrm{~Hz}, \mathrm{CH}_{2}, \mathrm{Az}\right), 28.6\left(\mathrm{~s}, \mathrm{CHCH}_{2} \mathrm{C}\right), 16.6\left(\mathrm{~s}, 4 \mathrm{C}, \mathrm{CH}_{2} \mathrm{CH}_{3}\right) .{ }^{31} \mathrm{P}\left\{{ }^{1} \mathrm{H}\right\}$ NMR (162 MHz, $\left.\mathrm{CDCl}_{3}\right) \delta 18.1$ (s, 2P). HRMS (ESI/Q-TOF) m/z: [M+H] ${ }^{+}$Calcd for $\mathrm{C}_{20} \mathrm{H}_{33} \mathrm{~N}_{2} \mathrm{O}_{6} \mathrm{P}_{2}$ : 459.1814; found: 459.1814.

\section{Tetraethyl ((2-(4-(benzyloxy)benzyl)aziridin-1-yl)methylene)(S)-bis(phosphonate) (3e)}

The title compound (108 mg, $0.21 \mathrm{mmol}, 46 \%$ yield) was obtained as a colorless oil following the procedure described above ( $3 \mathrm{~h}$ stirring at $80^{\circ} \mathrm{C}$ ) from compound $\mathbf{2 e}(150 \mathrm{mg}, 0.44 \mathrm{mmol})$.

$\mathrm{R} f$ dichloromethane/MeOH (95/5, v/v): 0.25. ${ }^{1} \mathrm{H} \mathrm{NMR}\left(500 \mathrm{MHz}, \mathrm{CDCl}_{3}\right) \delta 7.44-7.40(\mathrm{~m}, 2 \mathrm{H}$, CHAr), 7.40 - 7.35 (m, 2H, CHAr), 7.34 - 7.29 (m, 1H, CHAr), 7.14 - 7.09 (m, 2H, CHAr), 6.92 $6.87(\mathrm{~m}, 2 \mathrm{H}, \mathrm{CHAr}), 5.03\left(\mathrm{~s}, 2 \mathrm{H}, \mathrm{OCH}_{2} \mathrm{Ph}\right), 4.32-4.17\left(\mathrm{~m}, 8 \mathrm{H}, 4 \mathrm{CH}_{2} \mathrm{CH}_{3}\right), 3.26(\mathrm{dd}, \mathrm{J}=14.2,3.6$ $\mathrm{Hz}, 1 \mathrm{H}, \mathrm{CHCH}_{2} \mathrm{Ph}$ ), 2.39 (t, J = 18.1 Hz, 1H, PCHP), 2.34 (dd, J = 14.2, 8.6 Hz, 1H, $\mathrm{CHCH}_{2} \mathrm{Ph}$ ), 2.06 - 1.97 (m, 1H, CHAz), 1.90 (d, J = 4.0 Hz, 1H, CH 2 Az), 1.70 (dd, J = 6.4, 1.1 Hz, 1H, CH $2, \mathrm{Az}), 1.37$ $\left(\mathrm{t}, \mathrm{J}=7.1 \mathrm{~Hz}, 6 \mathrm{H}, 2 \mathrm{CH}_{2} \mathrm{CH}_{3}\right), 1.36-1.33\left(\mathrm{~m}, 6 \mathrm{H}, 2 \mathrm{CH}_{2} \mathrm{CH}_{3}\right) .{ }^{13} \mathrm{C}\left\{{ }^{1} \mathrm{H}\right\} \mathrm{NMR}\left(126 \mathrm{MHz}, \mathrm{CDCl}_{3}\right) \delta$ 157.5 (s, OCAr), 137.3 (s, CAr), 131.1 (s, CAr), 130.0 (s, 2C, CHAr), 128.7 (s, 2C, CHAr), 128.0 (s, CHAr), 127.6 (s, 2C, CHAr), 115.0 (s, 2C, CHAr), 70.1 (s, $\mathrm{OCH}_{2} \mathrm{Ph}$ ), 64.91 (t, J = 150.0 Hz, 1C, PCHP), 63.5 - 62.9 (m, 4C, $\mathrm{CH}_{2} \mathrm{CH}_{3}$ ), 43.2 (d, J = 16.3 Hz, CHAz), 38.0 (s, $\left.\mathrm{CHCH}_{2} \mathrm{Ph}\right), 36.4$ (d, J = $\left.16.8 \mathrm{~Hz}, \mathrm{CH}_{2}, \mathrm{Az}\right), 16.6$ (s, 4C, $\left.\mathrm{CH}_{2} \mathrm{CH}_{3}\right) .{ }^{31} \mathrm{P}\left\{{ }^{1} \mathrm{H}\right\} \mathrm{NMR}\left(202 \mathrm{MHz}, \mathrm{CDCl}_{3}\right) \delta 18.0$ (s, 2P). HRMS (ESI/Q-TOF) m/z: [M+H] ${ }^{+}$Calcd for $\mathrm{C}_{25} \mathrm{H}_{38} \mathrm{NO}_{7} \mathrm{P}_{2}$ : 526.2124; found: 526.2131.

\section{(S)-Tetraethyl((2-(2-(1H-benzo[d]imidazol-1-yl)ethyl)aziridin-1-yl)methylene)}

\section{bis(phosphonate) (3f)}

The title compound (133 mg, $0.28 \mathrm{mmol}, 50 \%$ yield) was obtained as a colorless oil following the procedure described above (stirring for $7 \mathrm{~h}$ at $55^{\circ} \mathrm{C}$ ) from compound $2 \mathbf{f}(162 \mathrm{mg}, 0.56 \mathrm{mmol})$.

$\mathrm{R} f$ dichloromethane/MeOH (95/5, v/v): 0.25. ${ }^{1} \mathrm{H} \mathrm{NMR}\left(400 \mathrm{MHz}, \mathrm{CDCl}_{3}\right) \delta 8.01$ (s, $\left.1 \mathrm{H}, \mathrm{NCHArN}\right)$, $7.80(\mathrm{dd}, \mathrm{J}=5.9,3.0 \mathrm{~Hz}, 1 \mathrm{H}, \mathrm{HAr}), 7.41$ (dd, J = 5.9, $2.9 \mathrm{~Hz}, 1 \mathrm{H}, \mathrm{HAr}), 7.31-7.26$ (m, 2H, HAr), $4.51-4.34\left(\mathrm{~m}, 2 \mathrm{H}, \mathrm{CH}_{2} \mathrm{~N}\right), 4.31-4.15\left(\mathrm{~m}, 8 \mathrm{H}, 4 \mathrm{CH}_{2} \mathrm{CH}_{3}\right), 2.34$ (t, J = 18.1 Hz, 1H, PCHP), $2.19-$ $1.95\left(\mathrm{~m}, 1 \mathrm{H}, \mathrm{CH}_{2} \mathrm{CH}_{2} \mathrm{~N}\right), 1.92-1.86(\mathrm{~m}, 1 \mathrm{H}, \mathrm{CH}), 1.70\left(\mathrm{dd}, \mathrm{J}=9.6,5.2 \mathrm{~Hz}, 2 \mathrm{H}, \mathrm{CHCH}_{2} \mathrm{~N}\right), 1.35(\mathrm{t}, \mathrm{J}$ $\left.=7.1 \mathrm{~Hz}, 12 \mathrm{H}, 4 \mathrm{CH}_{3} \mathrm{CH}_{2}\right) .{ }^{13} \mathrm{C}\left\{{ }^{1} \mathrm{H}\right\} \mathrm{NMR}\left(151 \mathrm{MHz}, \mathrm{CDCl}_{3}\right) \delta 144.0$ (s, CAr), 143.3 (s, C-2), 133.8 
(s, CAr), 122.9 (s, CAr), 122.2 (s, CAr), 120.6 (s, CAr), 109.8 (s, CAr), 64.9 (t, J = 150.5 Hz, PCHP), $63.4\left(\mathrm{dd}, \mathrm{J}=26.4,6.4 \mathrm{~Hz}, 4 \mathrm{C}, \mathrm{CH}_{2} \mathrm{CH}_{3}\right), 42.8\left(\mathrm{~s}, \mathrm{CH}_{2} \mathrm{CH}_{2} \mathrm{~N}\right), 39.3$ (d, J = 20.5 Hz, CHN), 35.9 (d, J = $\left.15.0 \mathrm{~Hz}, \mathrm{CHCH}_{2} \mathrm{~N}\right), 33.4\left(\mathrm{~d}, \mathrm{~J}=40.2 \mathrm{~Hz}, \mathrm{CH}_{2} \mathrm{CH}_{2} \mathrm{~N}\right), 16.7$ (s, 4C, $\left.\mathrm{CH}_{3} \mathrm{CH}_{2}\right) .{ }^{31} \mathrm{P}\left\{{ }^{1} \mathrm{H}\right\} \mathrm{NMR}(162 \mathrm{MHz}$, $\left.\mathrm{CDCl}_{3}\right) \delta 17.8$ (s, 1P), 17.7 (s, 1P). HRMS (ESI/Q-TOF) m/z: [M+H] ${ }^{+}$Calcd for $\mathrm{C}_{20} \mathrm{H}_{34} \mathrm{~N}_{3} \mathrm{O}_{6} \mathrm{P}_{2}$ : 474.1923; found: 474.1932.

\section{Tetraethyl (((S)-2-((S)-sec-butyl)aziridin-1-yl)methylene)bis(phosphonate) (3g)}

The title compound (121 mg, $0.31 \mathrm{mmol}, 41 \%$ yield) was obtained as a colorless oil following the procedure described above (stirring for $2 \mathrm{~h}$ at $80^{\circ} \mathrm{C}$ ) from compound $2 \mathrm{~g}(150 \mathrm{mg}, 0.75 \mathrm{mmol})$.

$\mathrm{R} f$ petroleum ether/EtOAc (7/3, v/v): 0.10. ${ }^{1} \mathrm{H}$ NMR (500 MHz, MeOD) $\delta 4.31-4.15(\mathrm{~m}, 8 \mathrm{H}$, $4 \mathrm{CH}_{2} \mathrm{CH}_{3}$ ), 2.71 (t, J = 18.8 Hz, 1H, PCHP), 1.87 (tdd, J = 6.3, 4.5, 1.7 Hz, 1H, CHAz), $1.84-1.81$ (m, 1H, $\left.\mathrm{CH}_{2}, \mathrm{Az}\right), 1.76-1.67\left(\mathrm{~m}, 1 \mathrm{H}, \mathrm{CHCH}_{2} \mathrm{CH}_{3}\right), 1.66\left(\mathrm{dd}, \mathrm{J}=6.5,1.3 \mathrm{~Hz}, 1 \mathrm{H}, \mathrm{CH}_{2}, \mathrm{Az}\right), 1.44-$ $1.39\left(\mathrm{~m}, 1 \mathrm{H}, \mathrm{CHCH}_{3}\right), 1.37$ (tdd, $\left.\mathrm{J}=7.1,2.5,1.2 \mathrm{~Hz}, 12 \mathrm{H}, 4 \mathrm{CH}_{2} \mathrm{CH}_{3}\right), 1.31-1.19(\mathrm{~m}, 1 \mathrm{H}$, $\left.\mathrm{CHCH}_{2} \mathrm{CH}_{3}\right), 0.94\left(\mathrm{t}, \mathrm{J}=7.5 \mathrm{~Hz}, 3 \mathrm{H}, \mathrm{CH}_{3} \mathrm{CH}_{2} \mathrm{CH}\right), 0.81\left(\mathrm{~d}, \mathrm{~J}=6.9 \mathrm{~Hz}, 3 \mathrm{H}, \mathrm{CH}_{3} \mathrm{CH}\right) .{ }^{13} \mathrm{C}\left\{{ }^{1} \mathrm{H}\right\} \mathrm{NMR}$ (126 MHz, MeOD) $\delta 65.0(\mathrm{t}, \mathrm{J}=152.0 \mathrm{~Hz}, \mathrm{PCHP}), 64.6$ (ddd, $\mathrm{J}=17.5,13.8,6.8 \mathrm{~Hz}, 4 \mathrm{C}, \mathrm{CH}_{2} \mathrm{CH}_{3}$ ), $47.7(\mathrm{dd}, \mathrm{J}=13.7,5.2 \mathrm{~Hz}, \mathrm{CHAz}), 37.9\left(\mathrm{~s}, \mathrm{CHCH}_{3}\right), 35.3\left(\mathrm{~d}, \mathrm{~J}=14.6 \mathrm{~Hz}, \mathrm{CH}_{2} \mathrm{Az}\right), 28.6(\mathrm{~s}$, $\left.\mathrm{CHCH}_{2} \mathrm{CH}_{3}\right), 16.8\left(\mathrm{dd}, \mathrm{J}=10.8,4.8 \mathrm{~Hz}, 4 \mathrm{C}, \mathrm{CH}_{2} \mathrm{CH}_{3}\right), 14.9$ (s, $\left.\mathrm{CHCH}_{3}\right), 11.9$ (s, $\left.\mathrm{CHCH}_{2} \mathrm{CHy}\right)$. ${ }^{31} \mathrm{P}\left\{{ }^{1} \mathrm{H}\right\}$ NMR (202 MHz, MeOD) $\delta 18.2\left(\mathrm{~d}, \mathrm{~J}_{\mathrm{PP}}=5.1 \mathrm{~Hz}\right), 18.1\left(\mathrm{~d}, \mathrm{~J}_{\mathrm{PP}}=5.1 \mathrm{~Hz}\right) . \mathrm{HRMS}(\mathrm{ESI} / \mathrm{Q}-\mathrm{TOF})$ $\mathrm{m} / \mathrm{z}:[\mathrm{M}+\mathrm{H}]^{+}$Calcd for $\mathrm{C}_{15} \mathrm{H}_{34} \mathrm{NO}_{6} \mathrm{P}_{2}$ : 386.1861; found: 386.1860 .

\section{tert-Butyl (S)-2-(1-(bis(diethoxyphosphoryl)methyl)aziridin-2-yl)acetate (3h)}

The title compound (30 mg, $0.07 \mathrm{mmol}, 20 \%$ yield) was obtained as a colorless oil following the procedure described above (stirring for $2 \mathrm{~h}$ at room temperature) from compound $\mathbf{2 h}$ (100 $\mathrm{mg}, 0.34$ mmol).

$\mathrm{R} f$ dichloromethane/MeOH 95/5 (v/v): 0.22. ${ }^{1} \mathrm{H} \mathrm{NMR}\left(500 \mathrm{MHz}, \mathrm{CDCl}_{3}\right) \delta 4.30-4.16(\mathrm{~m}, 8 \mathrm{H}$, $\left.4 \mathrm{CH}_{2} \mathrm{CH}_{3}\right), 3.01\left(\mathrm{dd}, \mathrm{J}=15.9,3.1 \mathrm{~Hz}, 1 \mathrm{H}, \mathrm{CH}_{2} \mathrm{C}(\mathrm{O})\right), 2.38(\mathrm{t}, \mathrm{J}=18.1 \mathrm{~Hz}, 1 \mathrm{H}, \mathrm{PCHP}), 2.11-2.04$ (m, 1H, CHAz), 1.98 (dd, J = 15.9, $9.3 \mathrm{~Hz}, 1 \mathrm{H}, \mathrm{CH}_{2} \mathrm{C}(\mathrm{O})$ ), 1.95 (d, J = 3.8 Hz, 1H, CH $\left.2, \mathrm{Az}\right), 1.81$ (d, $\left.\mathrm{J}=6.5 \mathrm{~Hz}, 1 \mathrm{H}, \mathrm{CH}_{2}, \mathrm{Az}\right), 1.43\left(\mathrm{~s}, 9 \mathrm{H}, 3 \mathrm{CH}_{3}, \mathrm{tBu}\right), 1.39-1.31\left(\mathrm{~m}, 12 \mathrm{H}, 4 \mathrm{CH}_{2} \mathrm{CH}_{3}\right) .{ }^{13} \mathrm{C}\left\{{ }^{1} \mathrm{H}\right\} \mathrm{NMR}$ $\left(126 \mathrm{MHz} \mathrm{CDCl}_{3}\right) \delta 170.8(\mathrm{~s}, \mathrm{C}(\mathrm{O}) \mathrm{tBu}), 80.8\left(\mathrm{~s}, \mathrm{C}\left(\mathrm{CH}_{3}\right)_{3}, \mathrm{tBu}\right), 66.0-63.5(\mathrm{t}, \mathrm{J}=150.3 \mathrm{~Hz}, \mathrm{PCHP})$, $63.4-63.0\left(\mathrm{~m}, 4 \mathrm{C}, 4 \mathrm{CH}_{2} \mathrm{CH}_{3}\right), 39.0\left(\mathrm{~s}, \mathrm{CH}_{2} \mathrm{C}(\mathrm{O})\right), 38.4$ (d, J = 15.6 Hz, CHAz), 36.2 (d, J = 15.3 Hz, 2C, $\left.\mathrm{CH}_{2}, \mathrm{Az}\right), 28.2$ (s, 3C, 3CH $\left.3, \mathrm{tBu}\right), 16.6$ (s, 4C, 4CH $\mathrm{CH}_{2} \mathrm{CH}_{3} .{ }^{31} \mathrm{P}\left\{{ }^{1} \mathrm{H}\right\} \mathrm{NMR}\left(162 \mathrm{MHz}, \mathrm{CDCl}_{3}\right) \delta$ $17.8\left(\mathrm{~d}, \mathrm{~J}_{\mathrm{PP}}=3.3 \mathrm{~Hz}, 1 \mathrm{P}\right), 17.7\left(\mathrm{~d}, \mathrm{~J}_{\mathrm{PP}}=3.3 \mathrm{~Hz}, 1 \mathrm{P}\right)$. HRMS (ESI/Q-TOF) m/z: $[\mathrm{M}+\mathrm{H}]^{+}$Calcd for $\mathrm{C}_{17} \mathrm{H}_{36} \mathrm{NO}_{8} \mathrm{P}_{2}$ : 444.1911; found: 444.1913. 
tert-Butyl (S)-(4-(1-(bis(diethoxyphosphoryl)methyl)aziridin-2-yl)butyl)carbamate (3i)

The title compound (115 mg, $0.23 \mathrm{mmol}, 40 \%$ yield) was obtained as colorless oil following the procedure described above, (stirring for $4 \mathrm{~h}$ at room temperature) from compound $2 \mathbf{i}$ (200 $\mathrm{mg}, 0.58$ mmol).

$\mathrm{R} f$ dichloromethane/MeOH (95/5, v/v): 0.26. ${ }^{1} \mathrm{H}$ NMR (600 MHz, $\left.\mathrm{CDCl}_{3}\right) \delta 4.71(\mathrm{~s}, 1 \mathrm{H}, \mathrm{NH}), 4.31-$ $4.16\left(\mathrm{~m}, 8 \mathrm{H}, 4 \mathrm{CH}_{2} \mathrm{CH}_{3}\right), 3.15-3.06\left(\mathrm{~m}, 2 \mathrm{H}, \mathrm{NHCH}_{2}\right), 2.34$ (t, J = 18.2 Hz, 1H, PCHP), $1.83-1.79$ (m, 1H, CHAz), 1.79 (d, J = 4.3 Hz, 1H, CH2, Az), 1.66 (d, J = 6.4 Hz, 1H, $\left.\mathrm{CH}_{2}, \mathrm{Az}\right), 1.56-1.43$ (m, $\left.6 \mathrm{H}, 3 \mathrm{CH}_{2}\right), 1.43$ (s, 9H, $\left.\mathrm{CH}_{3}, \mathrm{Boc}\right), 1.39-1.34\left(\mathrm{~m}, 12 \mathrm{H}, 4 \mathrm{CH}_{2} \mathrm{CH}_{3}\right) .{ }^{13} \mathrm{C}\left\{{ }^{1} \mathrm{H}\right\} \mathrm{NMR}\left(151 \mathrm{MHz}, \mathrm{CDCl}_{3}\right)$ $\delta 156.2(\mathrm{~s}, \mathrm{C}(\mathrm{O}) \mathrm{Boc}), 65.3(\mathrm{t}, \mathrm{J}=150.3 \mathrm{~Hz}, \mathrm{PCHP}), 63.3-62.8\left(\mathrm{~m}, 4 \mathrm{C}, \mathrm{CH}_{2} \mathrm{CH}_{3}\right), 42.0(\mathrm{~d}, \mathrm{~J}=15.3$ $\mathrm{Hz}, \mathrm{CHAz}), 40.7$ (s, $\mathrm{CH}_{2} \mathrm{NH}$ ), 36.7 (d, J = 16.3 Hz, $\left.\mathrm{CH}_{2}, \mathrm{Az}\right), 29.9$ (s, 3C, 3CH $), 28.6$ (s, 3C, $\mathrm{CH}_{3}$, Boc), 18.0 - $15.6\left(\mathrm{~m}, 4 \mathrm{C}, \mathrm{CH}_{2} \mathrm{CH}_{3}\right) .{ }^{31} \mathrm{P}\left\{{ }^{1} \mathrm{H}\right\} \mathrm{NMR}\left(162 \mathrm{MHz}, \mathrm{CDCl}_{3}\right) \delta 18\left(\mathrm{~d}, \mathrm{~J}_{\mathrm{PP}}=3.2 \mathrm{~Hz}\right), 17.9$ (d, $\mathrm{J}_{\mathrm{PP}}=3.2 \mathrm{~Hz}$ ). HRMS (ESI/Q-TOF) m/z: $[\mathrm{M}+\mathrm{H}]^{+}$Calcd for $\mathrm{C}_{20} \mathrm{H}_{43} \mathrm{~N}_{2} \mathrm{O}_{8} \mathrm{P}_{2}$ : 501.2495; found: 501.2497.

\section{Tetraethyl (((1R,6S)-7-azabicyclo[4.1.0]heptan-7-yl)methylene)bis(phosphonate) (3k)}

The title compound (120 mg, $0.31 \mathrm{mmol}, 72 \%$ yield) was obtained as a colorless oil following the procedure described above (stirring for $2 \mathrm{~h}$ at room temperature) from compound $\mathbf{2 k}$ (100 $\mathrm{mg}, 0.43$ mmol).

$\mathrm{R} f$ dichloromethane/MeOH 95/5 (v/v): 0.25. ${ }^{1} \mathrm{H}$ NMR $\left(500 \mathrm{MHz}, \mathrm{CDCl}_{3}\right) \delta 4.32-4.16(\mathrm{~m}, 8 \mathrm{H}$, $4 \mathrm{CH}_{2} \mathrm{CH}_{3}$ ), 2.39 (t, J = 18.0 Hz, 1H, PCHP), $1.94-1.87$ (m, 4H, 2CH 2 , 2CHAz), $1.77-1.70$ (m, 2H, $\left.\mathrm{CH}_{2}\right), 1.35\left(\mathrm{td}, \mathrm{J}=7.1,5.9 \mathrm{~Hz}, 12 \mathrm{H}, 4 \mathrm{CH}_{2} \mathrm{CH}_{3}\right), 1.34-1.30\left(\mathrm{~m}, 2 \mathrm{H}, 2 \mathrm{CH}_{2}\right), 1.20-1.11\left(\mathrm{~m}, 2 \mathrm{H}, 2 \mathrm{CH}_{2}\right)$. ${ }^{13} \mathrm{C}\left\{{ }^{1} \mathrm{H}\right\} \mathrm{NMR}\left(126 \mathrm{MHz}, \mathrm{CDCl}_{3}\right) \delta 64.7(\mathrm{t}, \mathrm{J}=149.8 \mathrm{~Hz}, \mathrm{PCHP}), 63.5-62.4\left(\mathrm{~m}, 4 \mathrm{C}, 4 \mathrm{CH}_{2} \mathrm{CH}_{3}\right)$, $41.3(\mathrm{~d}, \mathrm{~J}=16.4 \mathrm{~Hz}, 2 \mathrm{C}, 2 \mathrm{CHAz}), 24.1\left(\mathrm{~s}, 2 \mathrm{C}, 2 \mathrm{CH}_{2}\right), 20.5$ (s, 2C, 2CH$), 17.0-16.3$ (m, 4C, $\left.{ }_{4} \mathrm{CH}_{2} \mathrm{CH}_{3}\right) .{ }^{31} \mathrm{P}\left\{{ }^{1} \mathrm{H}\right\}$ NMR (202 MHz, $\left.\mathrm{CDCl}_{3}\right) \delta 18.2$ (s, 2P). HRMS (ESI/Q-TOF) m/z: [M+H] ${ }^{+} \mathrm{Calcd}$ for $\mathrm{C}_{15} \mathrm{H}_{32} \mathrm{NO}_{6} \mathrm{P}_{2}$ : 384.1705; found: 384.1705.

\section{(1aR,6aS)-1,1a,6,6a-Tetrahydroindeno[1,2-b]azirine (31)}

The title compound (57.7 mg, $0.44 \mathrm{mmol}, 97 \%$ yield) was obtained as a solid following the procedure described above (overnight stirring at room temperature) starting from compound $2 \mathbf{l}(N-\mathrm{Cbz}, 120 \mathrm{mg}$, $0.45 \mathrm{mmol}$ ); or from compound $3 \mathrm{~m}$ ( $N$-Boc, $120 \mathrm{mg}, 0.52 \mathrm{mmol})$ ) with $84 \%$ yield (57 $\mathrm{mg}, 0.43 \mathrm{mmol})$. $\mathrm{R} f$ dichloromethane/MeOH (95/5, v/v): 0.50. ${ }^{1} \mathrm{H}$ NMR $\left(500 \mathrm{MHz}, \mathrm{CDCl}_{3}\right) \delta 7.50-7.45(\mathrm{~m}, 1 \mathrm{H}$, CHAr), 7.36 - 7.24 (m, 2H, CHAr), 7.22 (d, J = 7.5 Hz, 1H, CHAr), 6.84 (s, 1H, NH), 5.93 (d, J = 7.5 $\mathrm{Hz}, 1 \mathrm{H}, \mathrm{CH}), 4.64$ (t, J = 7.0 Hz, 1H, CH), 3.21 (dd, J = 17.1, $\left.6.5 \mathrm{~Hz}, 1 \mathrm{H}, \mathrm{CH}_{2}\right), 3.03$ (d, J = 17.0 Hz, $\left.1 \mathrm{H}, \mathrm{CH}_{2}\right) .{ }^{13} \mathrm{C}\left\{{ }^{1} \mathrm{H}\right\} \mathrm{NMR}\left(126 \mathrm{MHz}, \mathrm{CDCl}_{3}\right) \delta 140.9$ (s, CAr), 138.4 (s, CAr), 130.3 (s, CHAr), 127.8 (s, CHAr), 126.3 (s, CHAr), 125.7 (s, CHAr), 84.4 (s, CHCH), 55.3 (s, CHCH), 39.6 (s, $\mathrm{CH}_{2}$ ). HRMS (ESI/Q-TOF) m/z: [M+H] $]^{+}$Calcd for $\mathrm{C}_{9} \mathrm{H}_{10} \mathrm{~N}$ : 132.0813 ; found: 132.0814 . 
Supporting Information. Copies of ${ }^{1} \mathrm{H},{ }^{13} \mathrm{C}$ and eventually ${ }^{31} \mathrm{P}$ NMR spectra of all new compounds described in the experimental section, copies of ${ }^{1} \mathrm{H}$ NMR spectra for known compounds or intermediates. NMR comparative study for compounds $\mathbf{2 d - 3 d}$ and $\mathbf{2 g} / \mathbf{3 g}$.

\section{Acknowledgments}

We are grateful to the CNRS (National Scientific Research Council) for financial support

\section{REFERENCES}

(1) Akhtar, R.; Naqvi, S. A. R.; Zahoor, A. F.; Saleem, S. Nucleophilic Ring Opening Reactions of Aziridines. Mol. Divers. 2018, 22 (2), 447-501. https://doi.org/10.1007/s11030-018-98290 .

(2) Polat-Cakir, S.; Beksultanova, N.; Dogan, Ö. Synthesis of Functionalized Novel $\alpha$-Amino- $\beta$ Alkoxyphosphonates through Regioselective Ring Opening of Aziridine-2-Phosphonates. Helv. Chim. Acta 2019, 102 (11), e1900199. https://doi.org/10.1002/hlca.201900199.

(3) Palacios, F.; Ochoa de Retana, A. M.; Alonso, J. M. Reaction of 2H-Azirine Phosphine Oxide and -Phosphonates with Nucleophiles. Stereoselective Synthesis of Functionalized Aziridines and $\alpha$ - and $\beta$-Aminophosphorus Derivatives. J. Org. Chem. 2005, 70 (22), 8895-8901. https://doi.org/10.1021/jo051404i.

(4) Moonen, K.; Laureyn, I.; Stevens, C. V. Synthetic Methods for Azaheterocyclic Phosphonates and Their Biological Activity. Chem. Rev. 2004, 104 (12), 6177-6215. https://doi.org/10.1021/cr030451c.

(5) Stevens, C.; Verbeke, A.; De Kimpe, N. A Convenient Synthesis of Dialkyl [[2(Bromomethyl)Aziridin-1-Yl]Methyl]Phosphonates, New Heterocyclic BetaAzaphosphonates. Synlett 1998, (2) 180-182.

(6) Russell, R. G. G. Bisphosphonates: From Bench to Bedside. Ann. N. Y. Acad. Sci. 2006, 1068 (1), 367-401. https://doi.org/10.1196/annals.1346.041.

(7) Fleisch, H. Bisphosphonates: Pharmacology and Use in the Treatment of Tumour-Induced Hypercalcaemic and Metastatic Bone Disease. Drugs 1991, 42 (6), 919-944. https://doi.org/10.2165/00003495-199142060-00003.

(8) Rogers, M. J.; Crockett, J. C.; Coxon, F. P.; Mönkkönen, J. Biochemical and Molecular Mechanisms of Action of Bisphosphonates. Bone 2011, 49 (1), 34-41. https://doi.org/10.1016/j.bone.2010.11.008. 
(9) Fazil, M.; Baboota, S.; Sahni, J. K.; Ameeduzzafar, null; Ali, J. Bisphosphonates:

Therapeutics Potential and Recent Advances in Drug Delivery. Drug Deliv. 2015, 22 (1), 1-9. https://doi.org/10.3109/10717544.2013.870259.

(10) Aoun, S.; Bennour, H. A Novel Hydroxy-Bisphosphonic Acid Prodrug as a Candidate for the Delivery of Ibuprofen to Bone. Synth. Commun. 2019, 49 (24), 3412-3418. https://doi.org/10.1080/00397911.2019.1671454.

(11) Farrell, K. B.; Karpeisky, A.; Thamm, D. H.; Zinnen, S. Bisphosphonate Conjugation for Bone Specific Drug Targeting. Bone Rep. 2018, 9, 47-60. https://doi.org/10.1016/j.bonr.2018.06.007.

(12) Simoni, D.; Gebbia, N.; Invidiata, F. P.; Eleopra, M.; Marchetti, P.; Rondanin, R.; Baruchello, R.; Provera, S.; Marchioro, C.; Tolomeo, M.; Marinelli, L.; Limongelli, V.; Novellino, E.; Kwaasi, A.; Dunford, J.; Buccheri, S.; Caccamo, N.; Dieli, F. Design, Synthesis, and Biological Evaluation of Novel Aminobisphosphonates Possessing an in Vivo Antitumor Activity through a Gammadelta-T Lymphocytes-Mediated Activation Mechanism. J. Med. Chem. 2008, 51 (21), 6800-6807. https://doi.org/10.1021/jm801003y.

(13) Kavanagh, K. L.; Guo, K.; Dunford, J. E.; Wu, X.; Knapp, S.; Ebetino, F. H.; Rogers, M. J.; Russell, R. G. G.; Oppermann, U. The Molecular Mechanism of Nitrogen-Containing Bisphosphonates as Antiosteoporosis Drugs. Proc. Natl. Acad. Sci. 2006, 103 (20), 7829 7834. https://doi.org/10.1073/pnas.0601643103.

(14) Romanenko, V.; P. Kukhar, V. 1-Amino-1,1-Bisphosphonates. Fundamental Syntheses and New Developments. Ark. Arch. Org. Chem. 2012, (v), 127-166. https://doi.org/10.3998/ark.5550190.0013.411.

(15) Bálint, E.; Tajti, Á.; Dzielak, A.; Hägele, G.; Keglevich, G. Microwave-Assisted Synthesis of (Aminomethylene)Bisphosphine Oxides and (Aminomethylene)Bisphosphonates by a ThreeComponent Condensation. Beilstein J. Org. Chem. 2016, 12 (1), 1493-1502. https://doi.org/10.3762/bjoc.12.146.

(16) Dąbrowska, E.; Burzyńska, A.; Mucha, A.; Matczak-Jon, E.; Sawka-Dobrowolska, W.; Berlicki, Ł.; Kafarski, P. Insight into the Mechanism of Three Component Condensation Leading to Aminomethylenebisphosphonates. J. Organomet. Chem. 2009, 694 (23), 38063813. https://doi.org/10.1016/j.jorganchem.2009.07.025.

(17) Yokomatsu, T.; Yoshida, Y.; Nakabayashi, N.; Shibuya, S. Simple and Efficient Method for Preparation of Conformationally Constrained Aminomethylene Gem-Diphosphonate Derivatives via Beckmann Rearrangement. J. Org. Chem. 1994, 59 (24), 7562-7564. https://doi.org/10.1021/jo00103a070. 
(18) Wu, M.; Chen, R.; Huang, Y. Simple, Efficient and One-Pot Method for Synthesis of Aminomethylene Gem-Diphosphonic Acid Derivatives from Ketones via Beckmann Rearrangement. Synthesis 2004, 2004 (15), 2441-2444. https://doi.org/10.1055/s-2004831233.

(19) Qian, D. Q.; Shi, X. D.; Cao, R. Z.; Liu, L. Z. The Synthesis and Reactivity of AlkylAminosubstitutedmethylenediphosphonates. Heteroat. Chem. 1999, 10 (4), 271-276. https://doi.org/10.1002/(SICI)1098-1071(1999)10:4<271::AID-HC2>3.0.CO;2-E.

(20) Wang, A.-E.; Chang, Z.; Sun, W.-T.; Huang, P.-Q. General and Chemoselective Bisphosphonylation of Secondary and Tertiary Amides. Org. Lett. 2015, 17 (3), 732-735. https://doi.org/10.1021/acs.orglett.5b00004.

(21) Prishchenko, A. A.; Livantsov, M. V.; Novikova, O. P.; Livantsova, L. I.; Ershov, I. S.; Petrosyan, V. S. Synthesis of New Types of Aminomethylenediphosphorus-Containing Acids and Their Derivatives. Russ. J. Gen. Chem. 2015, 85 (2), 370-379. https://doi.org/10.1134/S1070363215020048.

(22) Способ получения =фениламинотетралкилдифосфонметана - SU 445675 http://patents.su/2-445675-sposob-polucheniya-fenilaminotetralkildifosfonmetana.html (accessed Jun 26, 2019).

(23) Goldeman, W.; Kluczyński, A.; Soroka, M. The Preparation of N-Substituted Aminomethylidenebisphosphonates and Their Tetraalkyl Esters via Reaction of Isonitriles with Trialkyl Phosphites and Hydrogen Chloride. Part 1. Tetrahedron Lett. 2012, 53 (39), 5290-5292. https://doi.org/10.1016/j.tetlet.2012.07.085.

(24) Kurzak, B.; Goldeman, W.; Szpak, M.; Matczak-Jon, E.; Kamecka, A. Synthesis of N-Methyl Alkylaminomethane-1,1-Diphosphonic Acids and Evaluation of Their Complex-Formation Abilities towards Copper(II). Polyhedron 2015, 85, 675-684. https://doi.org/10.1016/j.poly.2014.09.031.

(25) Kaboudin, B.; Esfandiari, H.; Moradi, A.; Kazemi, F.; Aoyama, H. ZnCl2-Mediated Double Addition of Dialkylphosphite to Nitriles for the Synthesis of 1-Aminobisphosphonates. J. Org. Chem. 2019, 84 (22), 14943-14948. https://doi.org/10.1021/acs.joc.9b02298.

(26) Cheviet, T.; Wein, S.; Bourchenin, G.; Lagacherie, M.; Périgaud, C.; Cerdan, R.; Peyrottes, S. $\beta$-Hydroxy- and $\beta$-Aminophosphonate Acyclonucleosides as Potent Inhibitors of Plasmodium Falciparum Growth. J. Med. Chem. 2020, 63 (15), 8069-8087. https://doi.org/10.1021/acs.jmedchem.0c00131. 
(27) Stamm, H.; Gerster, G. Reactions with Aziridines XXI The (Michaelis-)Arbusov Reaction with N-Acyl Aziridines and Other Amidoethylations at Phosphorus. Tetrahedron Lett. 1980, 21 (17), 1623-1626. https://doi.org/10.1016/S0040-4039(00)77769-0.

(28) Stamm, H.; Gerster, G.; Baumann, T. Reaktionen mit Aziridinen, 29. N-Acylierte bzw. Nsulfonierte 2-Aminoethylphosphonsäureester durch basenkatalysierte Amidoethylierung von Phosphorigsäurediestern mit aktivierten Aziridinen. Chem. Ber. 1983, 116 (8), 2936-2957. https://doi.org/10.1002/cber.19831160821.

(29) Jörres, M.; Schiffers, I.; Atodiresei, I.; Bolm, C. Asymmetric Michael Additions of $\alpha-$ Nitrocyclohexanone to Aryl Nitroalkenes Catalyzed by Natural Amino Acid-Derived Bifunctional Thioureas. Org. Lett. 2012, 14 (17), 4518-4521. https://doi.org/10.1021/o1302005f.

(30) Jung, M. E.; Yi, S. W. Synthesis of Threo- $\beta$-Aminoalcohols from Aminoaldehydes via Chelation-Controlled Additions. Total Synthesis of 1-Threo Sphingosine and Safingol. Tetrahedron Lett. 2012, 53 (32), 4216-4220. https://doi.org/10.1016/j.tetlet.2012.05.153.

(31) Aaseng, J. E.; Gautun, O. R. Synthesis of Substituted (S)-2-Aminotetralins via Ring-Opening of Aziridines Prepared from 1-Aspartic Acid $\beta$-Tert-Butyl Ester. Tetrahedron 2010, 66 (46), 8982-8991. https://doi.org/10.1016/j.tet.2010.09.025.

(32) McGhee, A.; Cochran, B. M.; Stenmark, T. A.; Michael, F. E. Stereoselective Synthesis of 2,5-Disubstituted Morpholines Using a Palladium-Catalyzed Hydroamination Reaction. Chem. Commun. 2013, 49 (60), 6800-6802. https://doi.org/10.1039/C3CC44117B.

(33) ChemAxon - Software Solutions and Services for Chemistry \& Biology https://chemaxon.com/ (accessed Jul 26, 2019).

(34) Cardillo, G.; Gentilucci, L.; Gianotti, M.; Tolomelli, A. Influence of Lewis Acids on the Regioselectivity of N-Boc-Aziridine-2-Carboxylate Microwave-Assisted Rearrangement. Synlett 2000, 2000 (9), 1309-1311. https://doi.org/10.1055/s-2000-7125.

(35) Tomasini, C.; Vecchione, A. Novel Synthesis of 4-Carboxymethyl 5-Alkyl/Aryl Oxazolidin-2Ones by Rearrangement of 2-Carboxymethyl 3-Alkyl/Aryl N-Tert-Butoxycarbonyl Aziridines. Org. Lett. 1999, 1 (13), 2153-2156. https://doi.org/10.1021/o19912251.

(36) Ferraris, D.; Drury, W. J.; Cox, C.; Lectka, T. “Orthogonal” Lewis Acids: Catalyzed Ring Opening and Rearrangement of Acylaziridines. J. Org. Chem. 1998, 63 (14), 4568-4569. https://doi.org/10.1021/jo980558d.

(37) Shtrumfs, B.; Chernyak, D.; Kums, I.; Kalvins, I.; Trapencieris, P. Unnatural Amino Acids. 2. Simple Method of Obtaining Esters of Aziridine-2-Carboxylic Acids by a Transesterification 
Reaction. Chem. Heterocycl. Compd. 2004, 40 (6), 725-733.

https://doi.org/10.1023/B:COHC.0000040767.96090.cc.

(38) Pachamuthu, K.; Schmidt, R. R. Straightforward Synthesis of Gem-Phosphonate-Phosphate Containing Compounds via One-Pot Reaction of Thioesters with Diethyl Phosphite. Chem. Commun. 2004, No. 9, 1078-1079. https://doi.org/10.1039/B401079E.

(39) Ruel, R.; Bouvier, J.-P.; Young, R. N. Single-Step Preparation of 1-Hydroxybisphosphonates via Addition of Dialkyl Phosphite Potassium Anions to Acid Chlorides. J. Org. Chem. 1995, 60 (16), 5209-5213. https://doi.org/10.1021/jo00121a044.

(40) Demir, A. S.; Reis, Ö.; İğdir, A. Ç.; Esiringü, İ.; Eymur, S. Generation of Acyl Anion Equivalents from Acylphosphonates via Phosphonate-Phosphate Rearrangement: A Highly Practical Method for Cross-Benzoin Reaction. J. Org. Chem. 2005, 70 (25), 10584-10587. https://doi.org/10.1021/jo051811u.

(41) Jackson, J. A.; Hammond, G. B.; Wiemer, D. F. Synthesis of .Alpha.-Phosphono Lactones and Esters through a Vinyl Phosphate-Phosphonate Rearrangement. J. Org. Chem. 1989, 54 (20), 4750-4754. https://doi.org/10.1021/jo00281a012.

(42) Kondoh, A.; Terada, M. Brønsted Base Catalyzed [2,3]-Wittig/Phospha-Brook Tandem Rearrangement Sequence. Org. Lett. 2013, 15 (17), 4568-4571.

https://doi.org/10.1021/ol402144q.

(43) Kondoh, A.; Aoki, T.; Terada, M. Intramolecular Cyclization of Alkynyl $\alpha$-Ketoanilide Utilizing [1,2]-Phospha-Brook Rearrangement Catalyzed by Phosphazene Base. Org. Lett. 2014, 16 (13), 3528-3531. https://doi.org/10.1021/ol501479t.

(44) Hayashi, M.; Nakamura, S. Catalytic Enantioselective Protonation of $\alpha$-Oxygenated Ester Enolates Prepared through Phospha-Brook Rearrangement. Angew. Chem. Int. Ed. 2011, 50 (10), 2249-2252. https://doi.org/10.1002/anie.201007568.

(45) Ranga, S.; Chakravarty, M.; Chatterjee, T.; Ghosal, S. Mechanistic Insights into N-BuLi Mediated Phospha-Brook Rearrangement. New J. Chem. 2019, 43 (25), 9886-9890. https://doi.org/10.1039/C9NJ01867K.

(46) Hammerschmidt, F.; Schmidt, S. The Phosphonate-Phosphate- and Phosphate-Phosphonate Rearrangement and Their Applications, 4. Deprotonation of Secondary Benzylic Phosphates — Configurationally Stabile Benzylic Carbanions with a Diethoxyphosphoryloxy Substituent and Their Rearrangement to Optically Active Tertiary A-Hydroxyphosphonates. Chem. Ber. 1996, 129, 1503-1508. https://doi.org/10.1002/cber.19961291217.

(47) Hammerschmidt, F.; Schmidt, S. Metallation of Phosphorylated Aliphatic Alcohols to Configurationally Stable $\alpha$-Oxyalkyllithium Compounds - Use of the Phosphoryl Group as an 
Activating Group and Electrophile. Eur. J. Org. Chem. 2000, 2000 (12), 2239-2245. https://doi.org/10.1002/1099-0690(200006)2000:12<2239::AID-EJOC2239>3.0.CO;2-V.

(48) Fitch, S. J.; Moedritzer, Kurt. NMR Study of the P-C(OH)-P to P-C-O-P Rearrangement: Tetraethyl 1-Hydroxyalkylidenediphosphonates. J. Am. Chem. Soc. 1962, 84 (10), 1876-1879. https://doi.org/10.1021/ja00869a022.

(49) Armarego, W. L. F.; Perrin, D. D. Purification of Laboratory Chemicals; Butterworth Heinemann, 1997.

(50) Jamookeeah, C. E.; Beadle, C. D.; Jackson, R. F. W.; Harrity, J. P. A. Investigation of a Flexible Enantiospecific Approach to Aziridines. J. Org. Chem. 2008, 73 (3), 1128-1130. https://doi.org/10.1021/jo7023637.

(51) Braga, A. L.; Schneider, P. H.; Paixão, M. W.; Deobald, A. M.; Peppe, C.; Bottega, D. P. Chiral Seleno-Amines from Indium Selenolates. A Straightforward Synthesis of Selenocysteine Derivatives. J. Org. Chem. 2006, 71 (11), 4305-4307. https://doi.org/10.1021/jo060286b.

(52) Aaseng, J. E.; Gautun, O. R. Synthesis of (S)-2-Amino-7-Methoxytetralin and Isoindolo[1,2a]Isoquinolinone Derivatives from 1-Aspartic Acid. Tetrahedron 2014, 70 (34), 5057-5063. https://doi.org/10.1016/j.tet.2014.06.008.

(53) Song, L.; Servajean, V.; Thierry, J. Aziridines Derived from Amino Acids as Synthons in Pseudopeptide Synthesis. Tetrahedron 2006, 62 (15), 3509-3516. https://doi.org/10.1016/j.tet.2006.02.003.

(54) Sultane, P. R.; Mete, T. B.; Bhat, R. G. A Convenient Protocol for the Deprotection of NBenzyloxycarbonyl (Cbz) and Benzyl Ester Groups. Tetrahedron Lett. 2015, 56 (16), $2067-$ 2070. https://doi.org/10.1016/j.tetlet.2015.02.131.

(55) Yeung, B. K. S.; Zou, B.; Rottmann, M.; Lakshminarayana, S. B.; Ang, S. H.; Leong, S. Y.; Tan, J.; Wong, J.; Keller-Maerki, S.; Fischli, C.; Goh, A.; Schmitt, E. K.; Krastel, P.; Francotte, E.; Kuhen, K.; Plouffe, D.; Henson, K.; Wagner, T.; Winzeler, E. A.; Petersen, F.; Brun, R.; Dartois, V.; Diagana, T. T.; Keller, T. H. Spirotetrahydro $\beta$-Carbolines (Spiroindolones): A New Class of Potent and Orally Efficacious Compounds for the Treatment of Malaria. J. Med. Chem. 2010, 53 (14), 5155-5164. https://doi.org/10.1021/jm100410f.

(56) Mukai, T.; Suganuma, N.; Soejima, K.; Sasaki, J.; Yamamoto, F.; Maeda, M. Synthesis of a $\beta$ Tetrapeptide Analog as a Mother Compound for the Development of Matrix Metalloproteinase-2-Imaging Agents. Chem. Pharm. Bull. (Tokyo) 2008, 56 (3), 260-265. https://doi.org/10.1248/cpb.56.260. 
(57) Cui, P.; McCalmont, W. F.; Tomsig, J. L.; Lynch, K. R.; Macdonald, T. L. $\alpha$ - and $\beta$ Substituted Phosphonate Analogs of LPA as Autotaxin Inhibitors. Bioorg. Med. Chem. 2008, 16 (5), 2212-2225. https://doi.org/10.1016/j.bmc.2007.11.078.

(58) Tague, A. J.; Putsathit, P.; Hammer, K. A.; Wales, S. M.; Knight, D. R.; Riley, T. V.; Keller, P. A.; Pyne, S. G. Cationic Biaryl 1,2,3-Triazolyl Peptidomimetic Amphiphiles: Synthesis, Antibacterial Evaluation and Preliminary Mechanism of Action Studies. Eur. J. Med. Chem. 2019, 168, 386-404. https://doi.org/10.1016/j.ejmech.2019.02.013.

(59) Haghshenas, P.; Gravel, M. Chemo- and Diastereoselective N-Heterocyclic CarbeneCatalyzed Cross-Benzoin Reactions Using N-Boc- $\alpha$-Amino Aldehydes. Org. Lett. 2016, 18 (18), 4518-4521. https://doi.org/10.1021/acs.orglett.6b02123.

(60) Luna, A.; Astorga, C.; Fülöp, F.; Gotor, V. Enzymatic Resolution of ( \pm )-Cis-2Aminocyclopentanol and ( \pm -Cis-2-Aminocyclohexanol. Tetrahedron Asymmetry 1998, 9 (24), 4483-4487. https://doi.org/10.1016/S0957-4166(98)00482-0.

(61) Qin, Y.; Wang, C.; Huang, Z.; Xiao, X.; Jiang, Y. Synthesis of Enantiopure TertButanesulfinamide from Tert-Butanesulfinyloxazolidinone. J. Org. Chem. 2004, 69 (24), 8533-8536. https://doi.org/10.1021/jo048576k. 